Groups Geom. Dyn. 6 (2012), 249-278

DOI $10.4171 / \mathrm{GGD} / 157$
Groups, Geometry, and Dynamics

(C) European Mathematical Society

\title{
The geometry of right-angled Artin subgroups of mapping class groups
}

\author{
Matt T. Clay ${ }^{1}$, Christopher J. Leininger ${ }^{2}$ and Johanna Mangahas ${ }^{3}$
}

\begin{abstract}
We describe sufficient conditions which guarantee that a finite set of mapping classes generate a right-angled Artin group quasi-isometrically embedded in the mapping class group. Moreover, under these conditions, the orbit map to Teichmüller space is a quasi-isometric embedding for both of the standard metrics. As a consequence, we produce infinitely many genus $h$ surfaces (for any $h$ at least 2) in the moduli space of genus $g$ surfaces (for any $g$ at least 3 ) for which the universal covers are quasi-isometrically embedded in the Teichmüller space.
\end{abstract}

Mathematics Subject Classification (2000). 57M07, 20F65.

Keywords. Right-angled Artin groups, mapping class groups, pseudo-Anosov, Teichmüller space, surface subgroups.

\section{Introduction}

Let $S$ denote a surface and $\operatorname{Mod}(S)$ its mapping class group. Given independent pseudo-Anosov mapping classes $f_{1}, \ldots, f_{n} \in \operatorname{Mod}(S)$, McCarthy [35] and Ivanov [21] proved that by passing to sufficiently high powers, these mapping classes generate a free subgroup. This is the primary ingredient in the proof that $\operatorname{Mod}(S)$ satisfies the "Tits alternative"; see also [14], [32] for quantitative versions of this. Farb and Mosher [13] defined a notion of convex cocompactness for subgroups of $\operatorname{Mod}(S)$ by way of analogy with Kleinian groups, and proved that $f_{1}, \ldots, f_{n}$ could be raised to sufficiently high powers to further guarantee that the subgroup they generate is convex cocompact; see also [37], [22], [18].

Given an arbitrary set of elements $f_{1}, \ldots, f_{n} \in \operatorname{Mod}(S)$, we cannot expect that they generate a free group upon raising to sufficiently high powers. However, Koberda [25] has recently proven that the powers do generate a subgroup of a right-angled Artin subgroup of $\operatorname{Mod}(S)$; see also [9], [12], [8] for partial results in this direction.

\footnotetext{
${ }^{1}$ Partially supported by NSF grant DMS-1006898.

${ }^{2}$ Partially supported by NSF grant DMS-0905748.

${ }^{3}$ Partially supported by NSF RTG grant 0602191.
} 
In this paper, we are interested in geometric properties of right-angled Artin subgroups of the mapping class group. As convex cocompact subgroups are necessarily Gromov hyperbolic, we must consider other geometric properties for non-free rightangled Artin subgroups of $\operatorname{Mod}(S)$. For example, Crisp and Wiest [12] produced quasi-isometric embeddings of certain right-angled Artin groups into braid groups (and hence also mapping class groups). In this paper we show that this is possible in much greater generality, and furthermore, one can often conclude even stronger geometric statements for the corresponding subgroups. Here we state our main theorem, and refer the reader to Section 2 for necessary terminology and a more precise statement (Theorem 2.2).

Theorem 1.1. Suppose that $f_{1}, \ldots, f_{n} \in \operatorname{Mod}(S)$ are fully supported on overlapping nonannular subsurfaces. Then after raising to sufficiently high powers, these elements generate a quasi-isometrically embedded right-angled Artin subgroup of $\operatorname{Mod}(S)$. Furthermore, the orbit map to the Teichmüller space is a quasi-isometric embedding for both of the standard metrics, namely the Teichmüller and Weil-Petersson metrics.

Remarks. (1) We note that for the second statement to hold, the assumption that the support of each $f_{i}$ is not an annulus is necessary. On the other hand, it seems likely that the homomorphism to $\operatorname{Mod}(S)$ is a quasi-isometry without this additional assumption.

(2) There are a number of other "natural" metrics on Teichmüller space besides the two we have mentioned; the Bergman metric, Carathéodory metric, McMullen metric, Kähler-Einstein metric, Ricci metric and perturbed Ricci metric. However, each of these is quasi-isometric to the Teichmüller metric (see [36], [42], [29], [30]), and so the conclusion of Theorem 1.1 also holds for any of these metrics.

In Section 6 we use the ideas from the proof of this theorem to describe the Thurston type of any element in the right-angled Artin subgroup of $\operatorname{Mod}(S)$ we construct, and we see that it is pseudo-Anosov on the largest possible subsurface. In particular, we describe exactly which elements are pseudo-Anosov on $S$; see Theorem 6.1.

The hypotheses in Theorem 1.1 are general enough to easily provide quasiisometric embeddings of any right-angled Artin group into some mapping class group (see the end of Section 2.4). In particular we have the following.

Corollary 1.2. Any right-angled Artin group admits a homomorphism to some mapping class group which is a quasi-isometric embedding, and for which the orbit map to Teichmüller space is a quasi-isometric embedding with respect to either of the standard metrics.

The fundamental group of a closed orientable surface (of genus $h \geq 2$ ) is called a (genus $h$ ) surface subgroup. Many right-angled Artin groups contain quasi-isometrically embedded surface subgroups; see [40], [11] (though the question of exactly 
which right-angled Artin groups contain surface subgroups is still open; see for example [16], [23], [24], [10], [39]). There are also constructions of surface subgroups of the mapping class group [1], [26], [15]. In [27], infinitely many nonconjugate surface subgroups were constructed with geometric properties akin to geometric finiteness in the setting of Kleinian groups. From an explicit version of Corollary 1.2, and the aforementioned examples of surface subgroups of right-angled Artin groups, we obtain the following. See Section 5 for the proof.

Corollary 1.3. For any closed surface $S$ of genus at least 3 and any $h \geq 2$, there exist infinitely many nonconjugate genus $h$ surface subgroups of $\operatorname{Mod}(S)$, each of which acts cocompactly on some quasi-isometrically embedded hyperbolic plane in the Teichmüller space $\mathcal{T}(S)$, with either of the standard metrics.

This corollary is in contrast to the work of Bowditch [5] who proves finiteness, for any fixed $h \geq 2$, for the number of conjugacy classes of genus $h$ surface subgroups of $\operatorname{Mod}(S)$ which are purely pseudo-Anosov (we note that surface subgroups of the mapping class group which arise as subgroups of right-angled Artin groups can never be purely pseudo-Anosov; see Proposition 7.1 below). While these surface subgroups are not purely pseudo-Anosov, by the corollary, they do have the closely related property that every nontrivial element has positive translation length on $\mathcal{T}(S)$.

Finally, we remark that while Bowditch's result mentioned above is an example of a kind of rank-1 phenomenon for $\operatorname{Mod}(S)$, our examples illustrate higher rank behavior. Specifically, we could compare our results with those of Wang [41], who finds infinitely many conjugacy classes of discrete, faithful representations of right-angled Artin groups (hence surface subgroups) into higher rank Lie groups. Furthermore, Long, Reid and Thistlethwaite [31], find infinitely many conjugacy classes of Zariski dense, purely semi-simple representations of a surface group into $S L(3, \mathbb{Z})$. In fact, these surface groups are very closely related to the ones we study, in the sense that every nontrivial element has positive translation length on the associated symmetric space.

1.1. Plan of the paper. We begin in Section 2 by setting up the relevant definitions and notation we will use throughout. The section ends with a more precise version of our main theorem (Theorem 2.2). In Section 3 we describe an alternative space on which $\operatorname{Mod}(S)$ acts, namely Masur and Minsky's graph of markings [34]. We also state the required distance formulas (Theorems 3.1, 3.2 and 3.3) which provide the coarse estimates for the distances in the desired spaces, $\operatorname{Mod}(S)$ and $\mathcal{T}(S)$, in terms of sums of "local distances" between pairs of markings. These local distances are precisely the subsurface distances, also described in this section.

The idea of the proof of Theorem 2.2 is as follows. The hypothesis implies that each of the generators of the right-angled Artin group corresponds to a mapping class which makes progress in some subsurface - that is, it contributes nontrivially to some local distance. A geodesic in the Cayley graph of the right-angled Artin group 
determines a sequence of mapping classes, each of which makes progress in some subsurface. We need only ensure that this progress accumulates (that is, we need to avoid cancellation of local distances). This is verified by Theorem 5.2, which relates a partial order on the set of syllables in a minimal length representative for an element of the right-angled Artin group (see Section 4) with the partial order from [3] on the set of subsurfaces "between" a marking and its image under the associated mapping class (see Section 3.4). The details of the proof of Theorem 5.2 are carried out in Section 5, followed by the proof of Theorem 2.2.

In Section 6 we find the Thurston type of each element in the right-angled Artin subgroups of $\operatorname{Mod}(S)$ we are considering. We show that by conjugating to use the minimal number of generators to represent the element, it will be pseudo-Anosov on the smallest subsurface filled by the supports of the generators. For this, we use Masur and Minsky's Bounded Geodesic Image Theorem [34] to prove that the element acts with positive translation distance on the curve complex of the appropriate subsurface.

We end with a discussion of surface subgroups and the proofs of Corollary 1.3 and Proposition 7.1.

Acknowledgements. We would like to thank Richard Kent, Alan Reid, Thomas Koberda and Jason Behrstock for helpful conversations, and Sergio Fenley for carefully reading and commenting on an earlier version of this paper. We would also like to thank the Hausdorff Research Institute for Mathematics in Bonn, Germany, for its hospitality while this work was being completed.

\section{Notation and terminology}

2.1. Quasi-isometries. Given $A \geq 1$ and $B \geq 0$, we write $x \stackrel{A . B}{=} y$ to mean

$$
\frac{y-B}{A} \leq x \leq A y+B
$$

If $\left(\mathfrak{X}_{1}, d_{1}\right)$ and $\left(\mathfrak{X}_{2}, d_{2}\right)$ are metric spaces and $A \geq 1, B \geq 0$, then an $(A, B)$ quasi-isometric embedding from $\mathfrak{X}_{1}$ to $\mathfrak{X}_{2}$ is a map

$$
F: \mathfrak{X}_{1} \rightarrow \mathfrak{X}_{2}
$$

with the property that for all $x, y \in \mathfrak{X}_{1}$, we have

$$
d_{1}(x, y) \stackrel{A . B}{\asymp} d_{2}(F(x), F(y)) .
$$

If $F$ is an ( $A, B)$-quasi-isometric embedding for some $A$ and $B$, then we will say that $F$ is a quasi-isometric embedding.

If $F: \mathfrak{X}_{1} \rightarrow \mathfrak{X}_{2}$ is a quasi-isometric embedding and there is a constant $D>0$ so that any point of $\mathfrak{X}_{2}$ is within $D$ of some point of $F\left(\mathfrak{X}_{1}\right)$, then $F$ is called a quasi-isometry. 
2.2. Right angled Artin groups. Let $\Gamma$ be a graph with vertex set $\left\{s_{1}, \ldots, s_{n}\right\}$. The associated right-angled Artin group $G=G(\Gamma)$ is defined to be the group with presentation

$$
\left.G=\left\langle s_{1}, \ldots, s_{n}\right|\left[s_{i}, s_{j}\right]=1 \text { if }\left\{s_{i}, s_{j}\right\} \text { is an edge of } \Gamma\right\rangle .
$$

We will always work with the word metric on $G$ with respect to this generating set, and will denote it $d_{G}$.

Examples of right-angled Artin groups are free groups and direct products of free groups (in particular, free abelian groups). A simple example of a right-angled Artin group which is neither free nor a product of free groups is $G(\Gamma)$ where $\Gamma$ is the cyclic graph with 5 vertices shown in Figure 1.

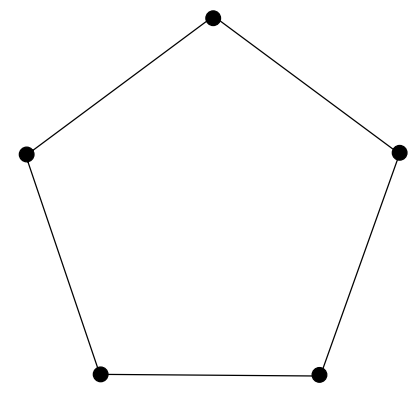

Figure 1. The cyclic graph with 5 vertices.

2.3. Surfaces. Given a connected surface $S$ of genus $g$ with $n$ punctures, the complexity is defined to be $\xi(S)=3 g-3+n$. Unless otherwise stated, we will assume throughout that $\xi(S)>0$. The mapping class group of $S$ is the group of isotopy classes of orientation preserving homeomorphisms of $S$ and is denoted $\operatorname{Mod}(S)$. By a curve in $S$, we mean the isotopy class of an essential (non-null-homotopic and non-peripheral) simple closed curve. A pants decomposition of $S$ is a maximal collection of distinct pairwise disjoint curves in $S$. Since $\xi(S)>0$, a nonempty pants decomposition exists and has precisely $\xi(S)$ curves in it.

A subsurface $X \subset S$ is essential if it is either a regular neighborhood of an essential simple closed curve, or else a component of the complement of an open regular neighborhood of a (possibly empty) union of pairwise disjoint essential simple closed curves. In particular, we assume that essential subsurfaces are connected. We will generally not distinguish between punctures and boundary components, and if $X \subset S$ has genus $h$ with $k$ punctures and $b$ boundary components, then we will write $\xi(X)=3 h-3+k+b$. Finally, we will assume that an essential subsurface $X$ has $\xi(X) \neq 0$, thus excluding a pair of pants as an essential subsurface. The set of all isotopy classes of essential subsurfaces $X$ of $S$ with $\xi(X) \neq 0$ will be denoted $\Omega(S)$.

We will often refer to the isotopy class of an essential subsurface simply as a subsurface. Furthermore, we will choose a nice representative for each curve and 


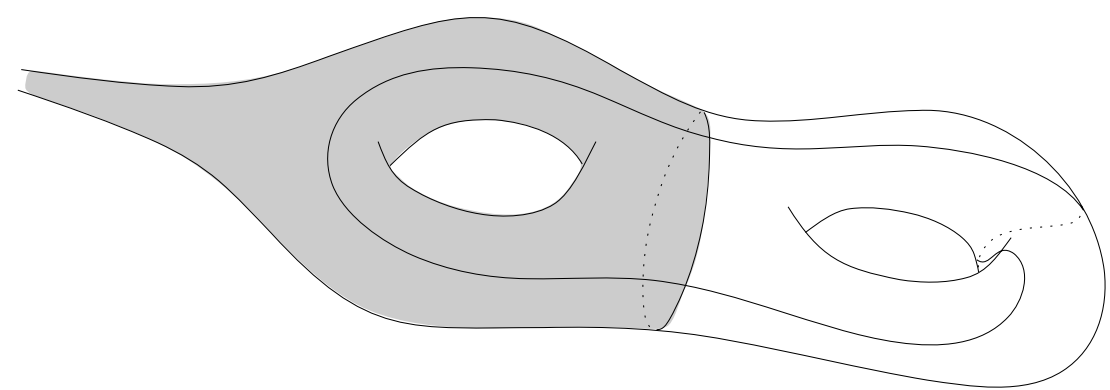

Figure 2. A genus 2 surface with 1 puncture $S$, a subsurface $X$ (shaded) and a curve $\gamma$.

each subsurface, and will not distinguish between a representative and its isotopy class when it is convenient. To be precise, we choose representatives as follows (annuli will play essentially no role in our discussion, so we do not bother describing their preferred representatives).

Fix a complete hyperbolic metric on $S$, and realize each curve by its unique geodesic representative. These representatives minimize the number of intersections (that is, they realize geometric intersection number). For each curve $\alpha$, we may choose some $\epsilon_{\alpha}$-neighborhood $N(\alpha)$ so that for any curves $\alpha$ and $\beta$, the intersections of $N(\alpha)$ and $N(\beta)$ correspond precisely to the intersections of $\alpha$ and $\beta$, and each such intersection is a "product square" (see Figure 4). For any nonannular subsurface $X$, which is a component of the complement of an open regular neighborhood of $\alpha_{1} \cup \cdots \cup \alpha_{k}$, we take its representative to be defined as the corresponding component of the complement of the interior of the neighborhood $N\left(\alpha_{1}\right) \cup \cdots \cup N\left(\alpha_{k}\right)$.

Suppose that $X, Y \subsetneq S$ are representative subsurfaces. Observe that $X \cap Y=\varnothing$ if and only if $X$ and $Y$ cannot be isotoped to be disjoint. If $X \cap Y \neq \emptyset, X \nsubseteq Y$ and $Y \nsubseteq X$, then we say that $X$ and $Y$ are overlapping, and write $X \pitchfork Y$.

One can check that this notion of overlapping agrees with that defined in [3], which is to say that $X \pitchfork Y$ if and only if some component of $\partial X$ cannot be isotoped disjoint from $Y$ and some component of $\partial Y$ cannot be isotoped disjoint from $X$.

2.4. Realizing a graph. Given a graph $\Gamma$, a surface $S$, and a collection of nonannular subsurfaces $X_{1}, \ldots, X_{n} \subset S$, we say that $\mathbb{X}=\left\{X_{1}, \ldots, X_{n}\right\}$ realizes $\Gamma$ nicely in $S$ if

(1) $X_{i} \cap X_{j}=\emptyset$ if and only if $\left\{s_{i}, s_{j}\right\}$ is an edge of $\Gamma$, and

(2) whenever $X_{i} \cap X_{j} \neq \emptyset$, then $X_{i} \pitchfork X_{j}$.

As Figure 3 indicates, there is a nice realization of the cyclic graph of length 5 in a genus 3 surface obtained from a branched cover of the sphere, branched over 8 points. By adding more points to this picture and taking a branched cover, we can produce nice realizations of this graph in any surface of genus $g \geq 3$. Moreover, given any graph it is easy to find some surface and a collection of subsurfaces which 
provide a nice realization (see [9], [11] for this kind of construction). We sketch one such construction here.

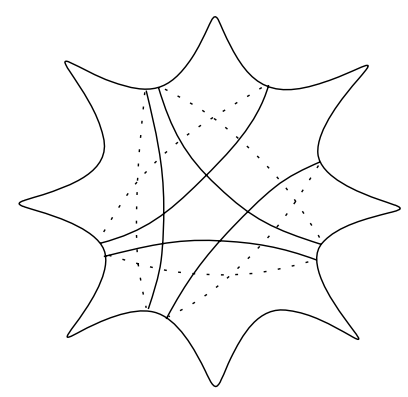

Figure 3. This figure represents a sphere with 8 punctures containing five curves, each of which bounds a disk with 3 punctures. These five 3-punctured disks provide a nonannular realization of the cyclic graph with 5 vertices. Taking a two-fold branched cover over the 8 points, we obtain a nonannular realization on a genus 3 surface (by 1-holed tori).

Starting with a graph $\Gamma$, we take a disjoint union of annuli, one for each vertex of $\Gamma$. Next, glue together the annuli along product squares whenever the associated vertices of $\Gamma$ are not connected by an edge. In each annulus, remove a disk and glue in a 1-holed torus. Finally, cap off the boundary components of the resulting surface with disks. See Figure 4 for a particular example.

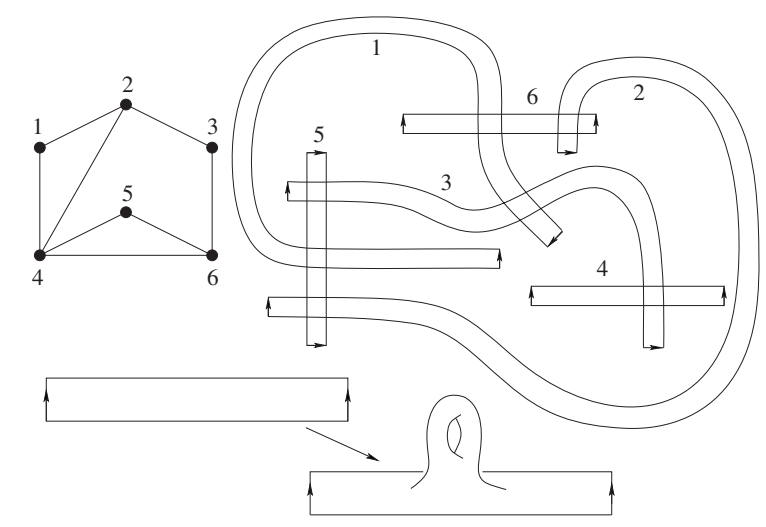

Figure 4. A graph $\Gamma$ and the associated annuli glued together along product squares as prescribed by $\Gamma$. At the bottom, we glue in a 1-holed torus to an annulus with a disk removed.

If $X$ is a nonannular subsurface of $S$ and $f \in \operatorname{Mod}(S)$ is the identity outside $X$, we say that $f$ is supported on $X$. We say that $f$ is fully supported on $X$ if we also have that $f$ is pseudo-Anosov on $X$. If $f$ is supported on $X$, then $f$ acts on $\mathcal{C}(X)$, the curve complex of $X$, and we let $\tau_{X}(f)$ denote the translation length of $f$ 
on $\mathcal{C}(X)$. This is defined by

$$
\tau_{X}(f)=\lim _{k \rightarrow \infty} \frac{d_{X}\left(\alpha, f^{k}(\alpha)\right)}{k},
$$

where $\alpha$ is any curve in $X$, and $d_{X}$ is the distance in $\mathscr{C}(X)$ - see Section 3.3. The following result of Masur and Minsky [33], Proposition 4.6, says that if $f$ is supported on $X$, then it is fully supported on $X$ if and only if $\tau_{X}(f)>0$.

Theorem 2.1 (Masur-Minsky). Given $X$ there exists $c>0$ so that if $f \in \operatorname{Mod}(X)$ is pseudo-Anosov, then

$$
d_{X}\left(\alpha, f^{k}(\alpha)\right) \geq c|k|
$$

for all $\alpha \in \mathcal{C}(X)$.

2.5. Homomorphisms. Suppose now that $\mathbb{X}=\left\{X_{1}, \ldots, X_{n}\right\}$ nicely realizes $\Gamma$ in $S$ and that $\mathbb{F}=\left\{f_{1}, \ldots, f_{n}\right\} \subset \operatorname{Mod}(S)$ are mapping classes. We say that $\mathbb{F}$ is (fully) supported on $\mathbb{X}$ if $f_{i}$ is (fully) supported on $X_{i}$ for each $i=1, \ldots, n$. Since homeomorphisms on disjoint subsurfaces commute, there is a unique homomorphism

$$
\phi_{\mathbb{F}}: G \rightarrow \operatorname{Mod}(S)
$$

defined by $\phi_{\mathbb{F}}\left(s_{i}\right)=f_{i}$.

We now state a more precise version of our main theorem. We write $\mathcal{T}(S)$ for the Teichmüller space, and we denote its two standard metrics by $d_{\mathcal{T}}$ for the Teichmüller metric and $d_{\mathrm{WP}}$ for the Weil-Petersson metric.

Theorem 2.2. Given a graph $\Gamma$ and a nice realization $\mathbb{X}=\left\{X_{1}, \ldots, X_{n}\right\}$ of $\Gamma$ in $S$, there exists a constant $C>0$ with the following property. If $\mathbb{F}=\left\{f_{1}, \ldots, f_{n}\right\}$ is fully supported on $\mathbb{X}$ and $\tau_{X_{i}}\left(f_{i}\right) \geq C$ for all $i=1, \ldots, n$, then the associated homomorphism

$$
\phi_{\mathbb{F}}: G(\Gamma) \rightarrow \operatorname{Mod}(S)
$$

is a quasi-isometric embedding. Furthermore, the orbit map $G \rightarrow \mathcal{T}(S)$ is a quasiisometric embedding for both $d_{\mathcal{T}}$ and $d_{\mathrm{WP}}$.

Remark. We reiterate for the casual reader that the subsurfaces $X_{i}$ are assumed to be essential, connected, and nonannular.

The proof of Theorem 2.2 will be carried out in Section 5. Theorem 2.2 easily implies Theorem 1.1.

\section{Projections and distance estimates}

Our proof of Theorem 2.2 uses results from [34], [6], [38] and [3]. The main construction we will use is that of subsurface projection, which we now briefly recall. 
3.1. Projections. Given a nonannular subsurface $X$ of $S$ and a curve $\gamma$, we define the projection of $\gamma$ to $X$, denoted $\pi_{X}(\gamma)$, to be the subset of $\mathcal{C}(X)$ constructed as follows. If $\gamma \cap X \neq \emptyset$, then either $\gamma$ is an essential simple closed curve in $X$, and we define $\pi_{X}(\gamma)=\{\gamma\}$, or else $\gamma \cap X$ is a disjoint union of essential arcs in $X$. For each $\operatorname{arc}$, consider $N$, the regular neighborhood of the union of this arc and the boundary components of $X$ which it meets. Then the boundary of $N$ is a union of curves in $X$ (and components of $\partial X$ ), and we define $\pi_{X}(\gamma)$ to be the set of all such curves in $X$, over all arcs of $\gamma \cap X$. See Figure 5.

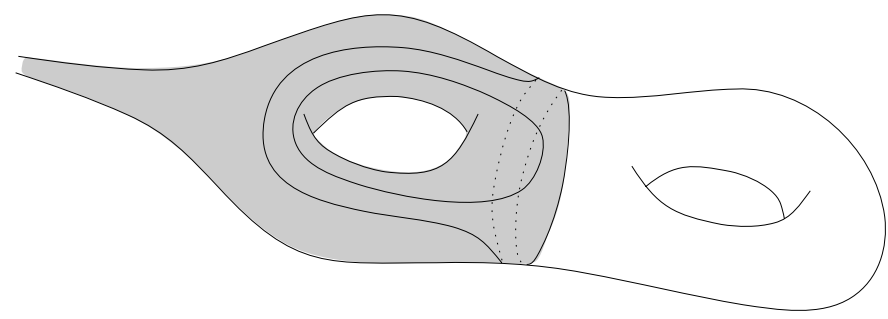

Figure 5. The projection $\pi_{X}(\gamma)$, where $S, X$ and $\gamma$ are as in Figure 2.

In general, the curves in $\pi_{X}(\gamma)$ need not be disjoint, but the set has diameter at most 2; see [34].

When $X$ is an annulus and $\gamma$ a curve, there is also a notion of a projection to $X$, which assigns to $\gamma$ a diameter one subset of the arc complex of $X$, denoted $\mathscr{C}(X)$, and again we denote this by $\pi_{X}(\gamma)$. For our purposes, simply the existence of this projection will suffice, so for the details of its definition, we refer the reader to [34].

If $\gamma$ is a disjoint union of curves $\gamma_{1} \cup \cdots \cup \gamma_{k}$, then we define $\pi_{X}(\gamma)$ to be the union $\bigcup_{i} \pi_{X}\left(\gamma_{i}\right)$. This set also has diameter at most 2. If $\gamma \cap X=\emptyset$, then $\pi_{X}(\gamma)=\emptyset$.

3.2. Markings. Another object we will need is a marking. For us, this will mean a complete clean marking in the sense of Masur and Minsky [34]. More precisely, a marking $\mu$ is a pants decomposition called the base of $\mu$

$$
\operatorname{base}(\mu)=\left\{\alpha_{1}, \ldots, \alpha_{\xi(S)}\right\},
$$

together with a transversal for each curve $\alpha_{i} \in \operatorname{base}(\mu)$ : this is a diameter at most one subset of $\mathcal{C}\left(X_{i}\right)$, where $X_{i}$ is the annular neighborhood of $\alpha_{i}$, together with some additional properties which we will not use explicitly; see [34] for a discussion.

Masur and Minsky [34] identify the set of all markings with the vertex set of a graph $\widetilde{\mathcal{M}}(S)$ called the marking graph of $S$. The edges of this graph correspond to certain elementary moves one can perform on a marking. We denote the resulting path metric on $\widetilde{\mathcal{M}}(S)$ by $d_{\tilde{\mathcal{M}}}$. The graph $\widetilde{\mathcal{M}}(S)$ is locally finite, and $\operatorname{Mod}(S)$ acts by isometries on it. In particular, the orbit map of this action is a quasi-isometry. We will use $\widetilde{\mathcal{M}}(S)$ as a model for $\operatorname{Mod}(S)$. 
Any marking $\mu$ can be projected to a subsurface. If $X$ is a nonannular subsurface, then $\pi_{X}(\mu)$ is defined to be $\pi_{X}(\operatorname{base}(\mu))$. For annuli, the projection is defined differently; see [34].

3.3. Distances. Given a subsurface $X$ and curves or markings $\mu$ and $\mu^{\prime}$, we define their distance in $X$ to be

$$
d_{X}\left(\mu, \mu^{\prime}\right)=\operatorname{diam}\left(\pi_{X}(\mu) \cup \pi_{X}\left(\mu^{\prime}\right)\right)
$$

where the diameter is computed in $\ell(X)$.

A trivial observation is that if $\mu, \mu^{\prime}$ are curves or markings on $S, f \in \operatorname{Mod}(S)$ is supported on $X$, and $Y$ is a nonannular subsurface disjoint from $X$ such that $\mu$ and $\mu^{\prime}$ have nonempty projection to $Y$, then

$$
d_{Y}\left(\mu, f\left(\mu^{\prime}\right)\right)=d_{Y}\left(\mu, \mu^{\prime}\right)
$$

Remark. We note that the validity of this observation relies on the assumption that $Y$ is nonannular.

Given $K>0$ and $\mu, \mu^{\prime} \in \tilde{\mathcal{M}}(S)$, define

$$
\Omega\left(K, \mu, \mu^{\prime}\right)=\left\{X \in \Omega(S) \mid d_{X}\left(\mu, \mu^{\prime}\right) \geq K\right\} .
$$

It is convenient to decompose $\Omega\left(K, \mu, \mu^{\prime}\right)$ into the annular subsurfaces $\Omega_{a}\left(K, \mu, \mu^{\prime}\right)$ and the nonannular subsurfaces $\Omega_{n}\left(K, \mu, \mu^{\prime}\right)$.

The following theorem is proven in [34].

Theorem 3.1 (Masur-Minsky). There exists $K_{0}>0$ (depending on $S$ ) so that if $K \geq K_{0}$, then there exist $A \geq 1, B \geq 0$ with the following property. For any $\mu, \mu^{\prime} \in \tilde{\mathcal{M}}(S)$ then

$$
d_{\widetilde{\mathcal{M}}}\left(\mu, \mu^{\prime}\right) \stackrel{A, B}{=} \sum_{X \in \Omega\left(K, \mu, \mu^{\prime}\right)} d_{X}\left(\mu, \mu^{\prime}\right) .
$$

A theorem of Brock [6] states that the Weil-Petersson metric on Teichmüller space is quasi-isometric to the pants graph. In [34], Masur and Minsky give a formula similar to that of the previous formula for distance in the pants graph. In particular combining these two results one obtains the following.

Theorem 3.2 (Brock, Masur-Minsky). There exist $K_{0}>0$ (depending on $S$ ) so that if $K \geq K_{0}$, then there exists $A \geq 1, B \geq 0$ with the following property. If $\mu, \mu^{\prime} \in \tilde{\mathcal{M}}(S)$ are shortest markings for $m, m^{\prime} \in \mathcal{T}(S)$, respectively, then

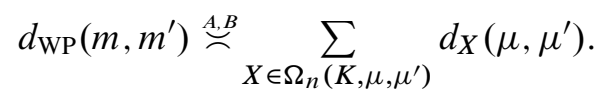


A shortest marking for $m$ is just a marking for which the pants decomposition has the shortest total length among all pants decompositions, and the transversals are projections of the shortest curves among those which can be used for transversals. For this theorem, the transversals are unimportant.

The analogous result for the Teichmüller metric was proven by Rafi in [38].

Theorem 3.3 (Rafi). There exists $K_{0}>0$ (depending on $S$ ) so that if $K \geq K_{0}$ and $\epsilon>0$ then there exist $A \geq 1, B \geq 0$ with the following property. If $\mu, \mu^{\prime} \in \widetilde{\mathcal{M}}(S)$ are shortest markings for $m, m^{\prime}$ in the $\epsilon$-thick part of $\mathcal{T}(S)$, respectively, then

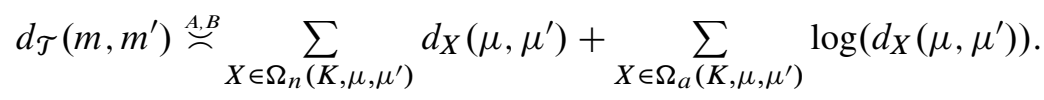

Remarks. (1) The special case of Theorem 2.2 in which $G(\Gamma)$ is abelian now follows immediately from the preceding three theorems and Theorem 2.1. Our proof is an extension of this idea.

(2) Strictly speaking, Theorems 3.1 and 3.2 would suffice for our purposes since, up to a constant, $d_{\mathrm{WP}}$ provides a lower bound for $d_{\mathcal{T}}$ by a result of Linch [28], and the lower bound on distortion is the only nontrivial inequality we need to prove. However, it seems worthwhile to include Theorem 3.3 as this illustrates a common interpretation for all of the metric spaces $\tilde{\mathcal{M}}(S)(\operatorname{or} \operatorname{Mod}(S)),\left(\mathcal{T}(S), d_{\mathcal{T}}\right)$, and $\left(\mathcal{T}(S), d_{\text {WP }}\right)$.

One final result about distances and subsurface projections which we will need is the following Bounded Geodesic Image Theorem [34].

Theorem 3.4 (Masur-Minsky). There exists $K_{0}>0$ (depending on $S$ ) so that if $\left\{v_{1}, \ldots, v_{n}\right\}$ is a geodesic in $\mathcal{C}(S)$ and $X \in \Omega(S)$, then either $\pi_{Y}\left(v_{j}\right)=\emptyset$ for some jor else

$$
\operatorname{diam}_{X}\left(\left\{\pi_{X}\left(v_{1}\right), \ldots, \pi_{X}\left(v_{n}\right)\right\}\right)<K_{0} .
$$

In particular, note that if $v, v^{\prime} \in \mathcal{C}(S)$ are two curves with $d_{X}\left(v, v^{\prime}\right) \geq K_{0}$, then any geodesic between $v$ and $v^{\prime}$ in $\digamma(S)$ must pass through a curve $v^{\prime \prime}$ disjoint from $X$ (for example, it may pass through a curve in $\partial X$ ).

For simplicity, we will assume, as we may, that $K_{0}$ is the same constant in all of the theorems in this section.

3.4. Partial order on subsurfaces. In [3], Behrstock, Kleiner, Minsky and Mosher defined a partial order on $\Omega\left(K, \mu, \mu^{\prime}\right)$ (for $K$ sufficiently large) which is closely related to the time-order constructed in [34] (see also [4]). However, as is noted in [3], while the time-order in [34] (which is defined on geodesics in hierarchies) requires a fair amount of the hierarchy machinery to describe it, the partial order on $\Omega\left(K, \mu, \mu^{\prime}\right)$ is completely elementary. As this is the basic tool we will use, we 
include the construction and verification of the necessary properties of this partial order, for the sake of completeness.

The starting point is the "Behrstock inequality" [2] (see also [32], Lemma 2.5, for the version stated here).

Proposition 3.5 (Behrstock). Suppose that $X$ and $Y$ are overlapping subsurfaces of $S$ and $\mu$ is a marking on $S$. Then

$$
d_{X}(\partial Y, \mu) \geq 10 \Longrightarrow d_{Y}(\partial X, \mu) \leq 4 .
$$

Suppose that $K \geq 20$. We define the partial order on $\Omega\left(K, \mu, \mu^{\prime}\right)$ as follows. Given $X, Y \in \Omega\left(K, \mu, \mu^{\prime}\right)$ with $X \pitchfork Y$, then we write $X \prec Y$ if

$$
d_{X}(\mu, \partial Y) \geq 10 .
$$

That this is a strict partial order is a consequence of the following useful description of $\prec$.

Proposition 3.6 (Behrstock-Kleiner-Minsky-Mosher). Suppose that $K \geq 20$ and $X, Y \in \Omega\left(K, \mu, \mu^{\prime}\right)$ with $X \pitchfork Y$. Then $X$ and $Y$ are ordered and the following are equivalent:

(2) $d_{X}(\mu, \partial Y) \geq 10$,

(5) $d_{Y}\left(\mu^{\prime}, \partial X\right) \geq 10$

(3) $d_{X}(\mu, \partial Y) \geq K-4$,

(6) $d_{Y}\left(\mu^{\prime}, \partial X\right) \geq K-4$,

(4) $d_{X}\left(\mu^{\prime}, \partial Y\right) \leq 4$,

(7) $\quad d_{Y}(\mu, \partial X) \leq 4$.

Proof. Assume the hypothesis of the proposition. Since $X \pitchfork Y$, we know that $\pi_{X}(\partial Y) \neq \emptyset$ and $\pi_{Y}(\partial X) \neq \emptyset$. To verify the equivalences, first observe that (1) and (2) are equivalent by definition, and since $K-4>10$, (3) implies (2) and (6) implies (5). Next, since $d_{X}\left(\mu, \mu^{\prime}\right), d_{Y}\left(\mu, \mu^{\prime}\right) \geq K$, the triangle inequality guarantees that (4) implies (3) and (7) implies (6). Furthermore, since $K-4>10$, Proposition 3.5 tells us that (2) implies (7) and (5) implies (4). This proves all the required implications.

Finally, we prove that $X$ and $Y$ are ordered. By the triangle inequality we have

$$
20 \leq K \leq d_{X}\left(\mu, \mu^{\prime}\right) \leq d_{X}(\mu, \partial Y)+d_{X}\left(\mu^{\prime}, \partial Y\right) .
$$

and so one of $d_{X}(\mu, \partial Y)$ or $d_{X}\left(\mu^{\prime}, \partial Y\right)$ is at least 10 . If $d_{X}(\mu, \partial Y) \geq 10$ then $X \prec Y$. If $d_{X}\left(\mu^{\prime}, \partial Y\right) \geq 10$, then reversing the roles of $X$ and $Y$ in each of the seven equivalent statements we see that $Y \prec X$, as required.

Corollary 3.7 (Behrstock-Kleiner-Minsky-Mosher). Suppose that $K \geq 20$. Then the relation $\prec i$ is a strict partial order. 
Proof. Since we never have $X \pitchfork X$, it follows that $\prec$ is non-reflexive. Furthermore, the equivalence of (2) and (7) in Proposition 3.6 means that $X \prec Y$ implies $Y \nprec X$, so $\prec$ is antisymmetric. Finally, if $X \prec Y$ and $Y \prec Z$ then we know $\pi_{Y}(\partial X)$ and $\pi_{Y}(\partial Z)$ are nonempty, and appealing to Proposition 3.6 and the triangle inequality we have

$20 \leq K \leq d_{Y}\left(\mu, \mu^{\prime}\right) \leq d_{Y}(\mu, \partial X)+d_{Y}(\partial X, \partial Z)+d_{Y}\left(\mu^{\prime}, \partial Z\right) \leq d_{Y}(\partial X, \partial Z)+8$

and so

$$
d_{Y}(\partial X, \partial Z) \geq 12>10 .
$$

In this case, $\partial X$ and $\partial Z$ intersect nontrivially in $Y$, so in particular, $X \pitchfork Z$.

Now we apply Proposition 3.5 to the preceding inequality to obtain

$$
d_{X}(\partial Y, \partial Z) \leq 4
$$

and hence by the triangle inequality

$$
16 \leq K-4 \leq d_{X}(\partial Y, \mu) \leq d_{X}(\partial Y, \partial Z)+d_{X}(\partial Z, \mu) \leq 4+d_{X}(\partial Z, \mu) .
$$

Therefore, $d_{X}(\partial Z, \mu) \geq 12>10$, and $X \prec Z$, proving transitivity.

\section{Normal forms in right-angled Artin groups}

Here we describe the normal forms in $G=G(\Gamma)$ as defined by Green [17], and Hermiller and Meier's procedure for obtaining these normal forms [19]. We refer the reader to Charney's survey article [7] for a discussion.

Suppose that $w=x_{1}^{e_{1}} \ldots x_{k}^{e_{k}}$ is a word in the generators $x_{i} \in\left\{s_{1}, \ldots, s_{n}\right\}$, where $e_{i} \in \mathbb{Z}$. Each $x_{i}^{e_{i}}$ is called a syllable of $w$. We consider the following moves which can be applied to $w$ (see also [20]):

(1) Remove a syllable $x_{i}^{e_{i}}$ if $e_{i}=0$.

(2) If $x_{i}=x_{i+1}$, then replace consecutive syllables $x_{i}^{e_{i}} x_{i+1}^{e_{i+1}}$ by $x_{i}^{e_{i}+e_{i+1}}$.

(3) If $\left[x_{i}, x_{i+1}\right]=1$, then replace $x_{i}^{e_{i}} x_{i+1}^{e_{i+1}}$ with $x_{i+1}^{e_{i+1}} x_{i}^{e_{i}}$.

Let $\operatorname{Min}(\sigma)$ be the set of words representing $\sigma \in G$ with the fewest number of syllables. Green's normal form for $\sigma$ is a certain type of element of $\operatorname{Min}(\sigma)$ obtained by stringing together, from left to right, maximal collections of commuting syllables. For us, we will consider any element of $\operatorname{Min}(\sigma)$ as a normal form, and we will shortly impose some additional structure on the set of syllables. First, we state the following from [19].

Theorem 4.1 (Hermiller-Meier). Any word representing $\sigma \in G$ can be transformed to any element of $\operatorname{Min}(\sigma)$ by applying a sequence of the moves above. In particular, in any such sequence, the number of syllables and the length does not increase. 
It follows that the words in $\operatorname{Min}(\sigma)$ determine geodesics in (the Cayley graph of) $G$ with respect to $s_{1}, \ldots, s_{n}$. Moreover, note that any two elements of $\operatorname{Min}(\sigma)$ differ by moves of type (3).

Let $w=x_{1}^{e_{1}} \ldots x_{k}^{e_{k}} \in \operatorname{Min}(\sigma)$ and consider the set of $\operatorname{syllables} \operatorname{syl}(w)=$ $\left\{x_{i}^{e_{i}}\right\}_{i=1}^{k}$. We consider this as a set of $k$ distinct elements: for example, we can artificially write this as $\left\{\left(x_{i}^{e_{i}}, i\right)\right\}_{i=1}^{k}$. If we have two elements $w, w^{\prime} \in \operatorname{Min}(\sigma)$ that differ by a single application of move (3) above, then there is an obvious bijection between $\operatorname{syl}(w)$ and syl $\left(w^{\prime}\right)$. Moreover, any sequence of these types of moves results in a sequence of bijections between the syllables of consecutive words in $\operatorname{Min}(\sigma)$. Observe that any such bijection between $\operatorname{syl}(w)$ and $\operatorname{syl}\left(w^{\prime}\right)$ sends a syllable of $w$ to one of $w^{\prime}$ representing the same element of $G$.

From this it follows that if any such sequence of moves ever brings a word $w$ back to itself, then the bijection from $\operatorname{syl}(w)$ to itself is the identity. The reason is that if $x_{i}^{e_{i}}$ and $x_{j}^{e_{j}}$ are syllables of $w$ which represent the same element in $G$ (so $x_{i}=x_{j}, e_{i}=e_{j}$ ), then if $x_{i}^{e_{i}}$ precedes $x_{j}^{e_{j}}$ in $w$, any of the bijections will preserve this property: a sequence of type (3) moves which would theoretically accomplish a swap of their positions, making $x_{j}^{e_{j}}$ precede $x_{i}^{e_{i}}$, would require a move where $x_{i}^{e_{i}}$ and $x_{j}^{e_{j}}$ are adjacent, at which time a type (2) move could be applied to reduce the number of syllables, and this is impossible. We use these bijections to identify the syllables of any two words $w, w^{\prime} \in \operatorname{Min}(\sigma)$, and simply write $\operatorname{syl}(\sigma)$ for this set of syllables.

We can define a strict partial order on $\operatorname{syl}(\sigma)$ by declaring $x_{i}^{e_{i}} \prec x_{j}^{e_{j}}$ if and only if $x_{i}^{e_{i}}$ precedes $x_{j}^{e_{j}}$ in every word $w \in \operatorname{Min}(\sigma)$. So for any $w \in \operatorname{Min}(\sigma)$, the order of the syllables is a refinement of the partial order (and the partial order is the largest partial order having this property for every $w \in \operatorname{Min}(\sigma))$.

\section{The proof of Theorem 2.1}

Throughout this section, we will assume $\mathbb{X}=\left\{X_{1}, \ldots, X_{n}\right\}$ realizes $\Gamma$ nicely in $S$, $\mathbb{F}=\left\{f_{1}, \ldots, f_{n}\right\}$ is fully supported on $\mathbb{X}$, and $\phi_{\mathbb{F}}: G=G(\Gamma) \rightarrow \operatorname{Mod}(S)$ is the associated homomorphism; see Sections 2.4 and 2.5 for notation.

Given a word $x_{1}^{e_{1}} \ldots x_{k}^{e_{k}}$ with $x_{i} \in\left\{s_{1}, \ldots, s_{n}\right\}$ for all $i$, let $J(i) \in\{1, \ldots, n\}$ be the unique number for which $x_{i}=s_{J(i)}$. For any $\sigma \in G$ and $w=x_{1}^{e_{1}} \ldots x_{k}^{e_{k}} \in$ $\operatorname{Min}(\sigma)$, set

$$
X^{w}\left(x_{i}^{e_{i}}\right)=\phi_{\mathbb{F}}\left(x_{1}^{e_{1}} \ldots x_{i-1}^{e_{i-1}}\right)\left(X_{J(i)}\right)
$$

for $i=2, \ldots, k$ and define $X^{w}\left(x_{1}^{e_{1}}\right)=X_{J(1)}$. We think of this as defining a map

$$
X^{w}: \operatorname{syl}(\sigma) \rightarrow \Omega(S) \text {. }
$$

Lemma 5.1. Suppose that $\Gamma, \mathbb{X}$ and $\mathbb{F}$ are as above. If $\sigma \in G(\Gamma)$ and $w, w^{\prime} \in$ $\operatorname{Min}(\sigma)$, then $X^{w}=X^{w^{\prime}}: \operatorname{syl}(\sigma) \rightarrow \Omega(S)$. 
Proof. Since any two words $w, w^{\prime} \in \operatorname{Min}(\sigma)$ differ by a sequence of moves of type (3), that is, in which adjacent commuting syllables are exchanged, it suffices to verify the lemma in the case that $w$ and $w^{\prime}$ differ by such a move:

$$
w=x_{1}^{e_{1}} \ldots x_{i}^{e_{i}} x_{i+1}^{e_{i+1}} \ldots x_{n}^{e_{n}} \quad \text { and } \quad w^{\prime}=x_{1}^{e_{1}} \ldots x_{i+1}^{e_{i+1}} x_{i}^{e_{i}} \ldots x_{n}^{e_{n}} .
$$

For $j \neq i$ or $i+1$, we clearly have $X^{w}\left(x_{j}^{e_{j}}\right)=X^{w^{\prime}}\left(x_{j}^{e_{j}}\right)$, and so we must show

$$
X^{w}\left(x_{i}^{e_{i}}\right)=X^{w^{\prime}}\left(x_{i}^{e_{i}}\right) \text { and } X^{w}\left(x_{i+1}^{e_{i+1}}\right)=X^{w^{\prime}}\left(x_{i+1}^{e_{i+1}}\right) .
$$

Interchanging the roles of $w$ and $w^{\prime}$, it suffices to prove just one of these equations, say $X^{w}\left(x_{i}^{e_{i}}\right)=X^{w^{\prime}}\left(x_{i}^{e_{i}}\right)$.

We have

$$
X^{w}\left(x_{i}^{e_{i}}\right)=\phi_{\mathbb{F}}\left(x_{1}^{e_{1}} \ldots x_{i-1}^{e_{i-1}}\right)\left(X_{J(i)}\right)
$$

whereas

$$
X^{w^{\prime}}\left(x_{i}^{e_{i}}\right)=\phi_{\mathbb{F}}\left(x_{1}^{e_{1}} \ldots x_{i-1}^{e_{i-1}} x_{i+1}^{e_{i+1}}\right)\left(X_{J(i)}\right)=\phi_{\mathbb{F}}\left(x_{1}^{e_{1}} \ldots x_{i-1}^{e_{i-1}}\right) \phi_{\mathbb{F}}\left(x_{i+1}^{e_{i+1}}\right)\left(X_{J(i)}\right) \text {. }
$$

Since $x_{i}^{e_{i}}$ and $x_{i+1}^{e_{i+1}}$ commute, $X_{J(i+1)}$, the support of $\phi_{\mathbb{F}}\left(x_{i+1}^{e_{i+1}}\right)=f_{J(i+1)}^{e_{i+1}}$, is disjoint from $X_{J(i)}$. Therefore,

$$
\phi_{\mathbb{F}}\left(x_{i+1}^{e_{i+1}}\right)\left(X_{J(i)}\right)=X_{J(i)},
$$

and the lemma follows.

By this lemma we can unambiguously define $X^{\sigma}=X^{w}$, independent of the choice of $w \in \operatorname{Min}(\sigma)$.

The main technical theorem we prove is the following. From this, together with Theorems 3.1, 3.2 and 3.3, our Theorem 2.2 (and hence also Theorem 1.1) follows easily.

Theorem 5.2. Suppose that $\Gamma$ and $\mathbb{X}$ are as above and $\mu \in \tilde{\mathcal{M}}(S)$. Then there exists a constant $K \geq K_{0}$ with the following property.

Suppose that $\mathbb{F}=\left\{f_{1}, \ldots, f_{n}\right\}$ is fully supported on $\mathbb{X}$ and that $\tau_{X_{j}}\left(f_{j}\right) \geq 2 K$ for all $1 \leq j \leq n$, and let $\phi_{\mathbb{F}}: G \rightarrow \operatorname{Mod}(S)$ be the associated homomorphism. Then, for any $\sigma \in G$ with $x_{1}^{e_{1}} \ldots x_{k}^{e_{k}} \in \operatorname{Min}(\sigma)$, we have:

(1) $d_{X^{\sigma}\left(x_{i}^{e_{i}}\right)}\left(\mu, \phi_{\mathbb{F}}(\sigma) \mu\right) \geq K\left|e_{i}\right|$ for each $i=1, \ldots, k$. Consequently,

$$
X^{\sigma}(\operatorname{syl}(\sigma)) \subset \Omega\left(K, \mu, \phi_{\mathbb{F}}(\sigma) \mu\right) .
$$

(2) $X^{\sigma}(\cdot): \operatorname{syl}(\sigma) \rightarrow \Omega\left(K, \mu, \phi_{\mathbb{F}}(\sigma) \mu\right)$ is an order-preserving injection.

Proof. Let

$$
K=K_{0}+20+2 \cdot \max \left\{d_{X_{j}}\left(\partial X_{i}, \mu\right) \mid i \neq j\right\} .
$$

Throughout the proof, we let $\phi=\phi_{\mathbb{F}}$.

In what follows, we prove statements (1) and (2) separately. For both, the proof is by induction on the number of syllables in $w \in \operatorname{Min}(\sigma)$. 
Proof of statement (1). To make the ideas in the proof more transparent, we introduce simplified notation. Given $w=x_{1}^{e_{1}} \ldots x_{k}^{e_{k}} \in \operatorname{Min}(\sigma)$, define

$$
g_{i}=\phi\left(x_{i}^{e_{i}}\right)=f_{J(i)}^{e_{i}} \quad \text { and } \quad Y_{i}=X_{J(i)} .
$$

Then $X^{\sigma}\left(x_{1}^{e_{1}}\right)=Y_{1}, X^{\sigma}\left(x_{2}^{e_{2}}\right)=g_{1} Y_{2}$, and in general $X^{\sigma}\left(x_{i}^{e_{i}}\right)=g_{1} g_{2} \ldots g_{i-1} Y_{i}$. In this notation, statement (1) claims that

$$
d_{g_{1} \ldots g_{i-1} Y_{i}}\left(\mu, g_{1} \ldots g_{k} \mu\right) \geq K\left|e_{i}\right|
$$

for $i=2, \ldots, k$, and also $d_{Y_{1}}\left(\mu, g_{1} \ldots g_{k} \mu\right) \geq K\left|e_{1}\right|$.

Suppose that $w$ has only one syllable. The claim only states that $d_{Y_{1}}\left(\mu, g_{1} \mu\right) \geq$ $K\left|e_{1}\right|$, which holds because, letting $j=J(1)$, we know

$$
\begin{aligned}
d_{Y_{1}}\left(\mu, g_{1} \mu\right) & =d_{X_{j}}\left(\mu, f_{j}^{e_{1}}(\mu)\right) \\
& =\operatorname{diam}_{X_{j}}\left(\pi_{X_{j}}(\mu) \cup \pi_{X_{j}}\left(\left(f_{j}^{e_{1}}\right) \mu\right)\right) \\
& =\operatorname{diam}_{X_{j}}\left(\pi_{X_{j}}(\mu) \cup f_{j}^{e_{1}}\left(\pi_{X_{j}}(\mu)\right)\right) \\
& \geq \tau_{X_{j}}\left(f_{j}^{e_{1}}\right) \\
& \geq 2 K\left|e_{1}\right| .
\end{aligned}
$$

Now suppose we have proved the claim for elements of $G=G(\Gamma)$ whose minimal representatives have at most $k-1$ syllables. Let $\sigma \in G$ with $w=x_{1}^{e_{1}} \ldots x_{k}^{e_{k}} \in$ $\operatorname{Min}(\sigma)$ having $k$ syllables. Define $g_{j}$ and $Y_{j}$ as above. Fix any $1 \leq i \leq k$ and separate the product $g_{1} \ldots g_{k}$ into subproducts, as illustrated below, with the additional possibility that $a, b$, or $c$ might be the empty word:

$$
\overbrace{g_{1} \ldots g_{\ell}}^{a} \overbrace{g_{\ell+1} \ldots g_{i-1}}^{b} g_{i} \overbrace{g_{i+1} \ldots g_{k}}^{c} .
$$

The subproducts $a, b$, and $c$ are defined as follows. By Lemma 5.1, we may assume that either $g_{i}$ and $g_{i+1}$ fail to commute, or by replacing $w$ with another word in $\operatorname{Min}(\sigma)$, that $i=k$. In the first case, let $c=g_{i+1} \ldots g_{k}$; in the latter case, let $c$ be the empty word. If there exists some syllable to the left of $g_{i}$ which does not commute with $g_{i}$, let $\ell<i$ be the largest index such that $g_{i}$ and $g_{\ell}$ do not commute, and let $a=g_{1} \ldots g_{\ell}$. Otherwise let $a$ be the empty word. Let $b=g_{\ell+1} \ldots g_{i-1}$ if $\ell+1<i$, and otherwise let $b$ be the empty word; observe that by construction, $b$ commutes with $g_{i}$.

Because $g_{1} \ldots g_{k}=a b g_{i} c$, we have

$$
d_{g_{1} \ldots g_{i-1} Y_{i}}\left(\mu, g_{1} \ldots g_{k} \mu\right)=d_{a b Y_{i}}\left(\mu, a b g_{i} c \mu\right)=d_{Y_{i}}\left(b^{-1} a^{-1} \mu, g_{i} c \mu\right) \text {. }
$$

By the triangle inequality and the fact that $d_{Y_{i}}\left(g_{i} c \mu, c \mu\right) \geq 2 K\left|e_{i}\right|$,

$$
d_{Y_{i}}\left(b^{-1} a^{-1} \mu, g_{i} c \mu\right) \geq 2 K\left|e_{i}\right|-d_{Y_{i}}\left(b^{-1} a^{-1} \mu, c \mu\right) .
$$


To control the last term we again employ the triangle inequality:

$$
d_{Y_{i}}\left(b^{-1} a^{-1} \mu, c \mu\right) \leq d_{Y_{i}}\left(b^{-1} a^{-1} \mu, \mu\right)+d_{Y_{i}}(\mu, c \mu) .
$$

Because $b$ is the (possibly empty) product of syllables $g_{j}$ that commute with $g_{i}$, $b$ acts as the identity on $Y_{i}$. Therefore we have

$$
\begin{aligned}
d_{Y_{i}}\left(b^{-1} a^{-1} \mu, \mu\right) & =d_{Y_{i}}\left(a^{-1} \mu, b \mu\right) \\
& =\operatorname{diam}_{Y_{i}}\left(\pi_{Y_{i}}\left(a^{-1} \mu\right) \cup \pi_{Y_{i}}(b \mu)\right) \\
& =\operatorname{diam}_{Y_{i}}\left(\pi_{Y_{i}}\left(a^{-1} \mu\right) \cup \pi_{Y_{i}}(\mu)\right) \\
& =d_{Y_{i}}\left(a^{-1} \mu, \mu\right) .
\end{aligned}
$$

So far we have shown

$$
d_{g_{1} \ldots g_{i-1} Y_{i}}\left(\mu, g_{1} \ldots g_{k} \mu\right) \geq 2 K\left|e_{i}\right|-d_{Y_{i}}\left(a^{-1} \mu, \mu\right)-d_{Y_{i}}(\mu, c \mu) .
$$

To finish, we prove that the last two terms on the right are each less than $K / 2$. Since the signs of the $e_{i}$ never come into play, the proof is very similar for either term, so we focus on $d_{Y_{i}}(\mu, c \mu)$. If $c$ is the empty word, then $d_{Y_{i}}(\mu, c \mu)=\operatorname{diam}_{Y_{i}}(\mu) \leq$ $2 \leq K / 2$. Otherwise $c=g_{i+1} \ldots g_{k}$. Because subwords of minimal words are also minimal, $c=\phi\left(\sigma_{c}\right)$ for some $\sigma_{c} \in G$ with minimal word $x_{i+1}^{e_{i}+1} \ldots x_{k}^{e_{k}}$, which has strictly less than $k$ syllables. Let $x_{1}^{\prime}=x_{i+1}^{e_{i}+1}$ be the first syllable. Applying the induction hypothesis, we have

$$
d_{Y_{i+1}}\left(\mu, g_{i+1} \ldots g_{k} \mu\right)=d_{Y_{i+1}}(\mu, c \mu)=d_{X^{\sigma_{c}\left(x_{1}^{\prime}\right)}}\left(\mu, \phi\left(\sigma_{c}\right) \mu\right) \geq K\left|e_{i+1}\right| .
$$

By our choice of $K, d_{Y_{i+1}}\left(\mu, \partial Y_{i}\right)=d_{X_{J(i+1)}}\left(\mu, \partial X_{J(i)}\right) \leq K / 2$. Since $g_{i}$ and $g_{i+1}$ do not commute, $Y_{i} \pitchfork Y_{i+1}$, so we may apply the triangle inequality to obtain

$$
\begin{aligned}
d_{Y_{i+1}}\left(\partial Y_{i}, g_{i+1} \ldots g_{k} \mu\right) & \geq K\left|e_{i+1}\right|-d_{Y_{i+1}}\left(\mu, \partial Y_{i}\right) \\
& \geq K\left|e_{i+1}\right|-K / 2 \geq K / 2 \geq 20 / 2=10 .
\end{aligned}
$$

Appealing to Proposition 3.5, we know that $d_{Y_{i}}\left(\partial Y_{i+1}, g_{i+1} \ldots g_{k} \mu\right) \leq 4$. By our choice of $K, d_{Y_{i}}\left(\mu, \partial Y_{i+1}\right)=d_{X_{J(i)}}\left(\mu, \partial X_{J(i+1)}\right) \leq K / 2-4$, so combining, we have

$$
\begin{aligned}
d_{Y_{i}}(\mu, c \mu) & \leq d_{Y_{i}}\left(\mu, \partial Y_{i+1}\right)+d_{Y_{i}}\left(\partial Y_{i+1}, c \mu\right) \\
& =d_{Y_{i}}\left(\mu, \partial Y_{i+1}\right)+d_{Y_{i}}\left(\partial Y_{i+1}, g_{i+1} \ldots g_{k} \mu\right) \\
& \leq K / 2-4+4=K / 2 .
\end{aligned}
$$

The entire argument can be mirrored for $d_{Y_{i}}\left(a^{-1} \mu, \mu\right)$, starting with the observation that either $a^{-1}$ is the empty word or $a^{-1}=g_{\ell}^{-1} \ldots g_{1}^{-1}$, where $g_{\ell}$ and $g_{i}$ do not commute.

To summarize, we have shown

$$
d_{g_{1} \ldots g_{i-1} Y_{i}}\left(\mu, g_{1} \ldots g_{k} \mu\right) \geq 2 K\left|e_{i}\right|-K / 2-K / 2 \geq K\left|e_{i}\right|,
$$

completing the induction for statement (1). 
Proof of statement (2). We will now show that $X^{\sigma}$ is an order-preserving injection. The base case for the induction is when $\sigma$ has one syllable, and then the conclusion is trivially verified. We assume that the conclusion holds for elements $\sigma$ with at most $k-1$ syllables, and prove that it also holds for elements with $k$ syllables.

The subwords $w_{\text {init }}=x_{1}^{e_{1}} \ldots x_{k-1}^{e_{k-1}}$ and $w_{\text {term }}=x_{2}^{e_{2}} \ldots x_{k}^{e_{k}}$ of $w$ are clearly minimal representatives of the elements $\sigma_{\text {init }}, \sigma_{\text {term }} \in G$ they represent. Furthermore, the partial order on the syllables of $w_{\text {init }}$ and $w_{\text {term }}$ is the restriction of the partial order on the syllables of $w$.

By the inductive hypothesis, the conclusion of the theorem holds for $\sigma_{\text {init }}$ and $\sigma_{\text {term }}$. By construction we have

$$
X^{\sigma}\left(x_{i}^{e_{i}}\right)= \begin{cases}X^{\sigma_{\text {init }}}\left(x_{i}^{e_{i}}\right) & \text { if } i \neq k, \\ \phi\left(x_{1}^{e_{1}}\right)\left(X^{\sigma_{\mathrm{term}}}\left(x_{i}^{e_{i}}\right)\right) & \text { if } i \neq 1 .\end{cases}
$$

If $i$ is neither 1 nor $k$, then the two defining expressions are indeed equal. In particular, notice that to establish injectivity, we need only show that $X^{\sigma}\left(x_{1}^{e_{1}}\right) \neq X^{\sigma}\left(x_{k}^{e_{k}}\right)$.

Suppose that $x_{i}^{e_{i}} \prec x_{j}^{e_{j}}$ for two syllables of $\sigma$. If $j \neq k$, then both are syllables of $\sigma_{\text {init }}$ and hence by induction $X^{\sigma_{\text {init }}}\left(x_{i}^{e_{i}}\right) \prec X^{\sigma_{\text {init }}}\left(x_{j}^{e_{j}}\right)$. Thus $X^{\sigma_{\text {init }}}\left(x_{i}^{e_{i}}\right) \pitchfork X^{\sigma_{\text {init }}}\left(x_{j}^{e_{j}}\right)$ and

$$
d_{X^{\sigma_{\text {init }}}\left(x_{i}^{e_{i}}\right)}\left(\mu, \partial X^{\sigma_{\text {init }}}\left(x_{j}^{e_{j}}\right)\right) \geq 10 .
$$

Since $X^{\sigma_{\text {init }}}\left(x_{i}^{e_{i}}\right)=X^{\sigma}\left(x_{i}^{e_{i}}\right)$ and $X^{\sigma_{\text {init }}}\left(x_{j}^{e_{j}}\right)=X^{\sigma}\left(x_{j}^{e_{j}}\right)$, we have $X^{\sigma}\left(x_{i}^{e_{i}}\right) \prec$ $X^{\sigma}\left(x_{j}^{e_{j}}\right)$. If $i \neq 1$, then we can make a similar argument using the induction hypothesis applied to $\sigma_{\mathrm{term}}$. In this case we appeal to condition (4) of Proposition 3.6 to conclude that

$$
d_{X^{\sigma_{\mathrm{term}}}\left(x_{i}^{e_{i}}\right)}\left(\partial X^{\sigma_{\mathrm{term}}}\left(x_{j}^{e_{j}}\right), \phi\left(\sigma_{\mathrm{term}}\right) \mu\right) \leq 4,
$$

so that, applying $\phi\left(x_{1}^{e_{1}}\right)$, this becomes

$$
d_{X^{\sigma}\left(x_{i}^{e_{i}}\right)}\left(\partial X^{\sigma}\left(x_{j}^{e_{j}}\right), \phi(\sigma) \mu\right) \leq 4,
$$

and so $X^{\sigma}\left(x_{i}^{e_{i}}\right) \prec X^{\sigma}\left(x_{j}^{e_{j}}\right)$, as required.

Now suppose $x_{1}^{e_{1}} \prec x_{k}^{e_{k}}$. There are two cases.

Case 1. There is a syllable $x_{i}^{e_{i}}$ such that $x_{1}^{e_{1}} \prec x_{i}^{e_{i}} \prec x_{k}^{e_{k}}$.

Arguing as above, by induction we have $X^{\sigma}\left(x_{1}^{e_{1}}\right) \prec X^{\sigma}\left(x_{i}^{e_{i}}\right)$ and $X^{\sigma}\left(x_{i}^{e_{i}}\right) \prec$ $X^{\sigma}\left(x_{k}^{e_{k}}\right)$ in $\Omega(K, \mu, \phi(\mu))$ and hence by Corollary 3.7 we have that $X^{\sigma}\left(x_{1}^{e_{1}}\right) \prec$ $X^{\sigma}\left(x_{k}^{e_{k}}\right)$.

Case 2. There is no syllable $x_{i}^{e_{i}}$ such that $x_{1}^{e_{1}} \prec x_{i}^{e_{i}} \prec x_{k}^{e_{k}}$.

Then there is a word $w \in \operatorname{Min}(\sigma)$ of the form

$$
w=w_{1} x_{1}^{e_{1}} x_{k}^{e_{k}} w_{2},
$$


where $\left[w_{1}, x_{1}\right]=1$ and $\left[x_{k}, w_{2}\right]=1$ (and either or both of $w_{i}$ may be the empty word). Now

$$
\begin{aligned}
d_{X_{J(1)}}\left(\mu, \partial X^{\sigma}\left(x_{k}^{e_{k}}\right)\right) & =d_{X_{J(1)}}\left(\mu, \phi\left(w_{1}\right) \phi\left(x_{1}^{e_{1}}\right) \partial X_{J(k)}\right) \\
& =d_{X_{J(1)}}\left(\mu, \phi\left(x_{1}^{e_{1}}\right) \partial X_{J(k)}\right) \\
& \geq d_{X_{J(1)}}\left(\mu, \phi\left(x_{1}^{e_{1}}\right) \mu\right)-d_{X_{J(1)}}\left(\phi\left(x_{1}^{e_{1}}\right) \mu, \phi\left(x_{1}^{e_{1}}\right) \partial X_{J(k)}\right) \\
& \geq 2 K\left|e_{1}\right|-(K-20) / 2 \geq 10,
\end{aligned}
$$

where the second equality comes from the fact that $w_{1}$ commutes with $x_{1}$, and so $\phi\left(w_{1}\right)$ is the identity on $X_{J(1)}$. Thus, by Proposition $3.6(2)$, we have $X^{\sigma}\left(x_{1}^{e_{1}}\right) \prec$ $X^{\sigma}\left(x_{k}^{e_{k}}\right)$.

In particular, if $x_{1}^{e_{1}} \prec x_{k}^{e_{k}}$ then $X^{\sigma}$ is injective.

All that remains now is to show that if $x_{1}^{e_{1}}$ and $x_{k}^{e_{k}}$ are not comparable by $\prec$, then $X^{\sigma}\left(x_{1}^{e_{1}}\right) \neq X^{\sigma}\left(x_{k}^{e_{k}}\right)$. If they are not comparable, then $\sigma$ is represented by a word of the form $w=w_{1} x_{1}^{e_{1}} x_{k}^{e_{k}} w_{2}$, where as above $\left[w_{1}, x_{1}\right]=1$ and $\left[x_{k}, w_{2}\right]=1$. Furthermore, $x_{1}^{e_{1}}$ and $x_{k}^{e_{k}}$ must commute, and hence it is clear that $X^{\sigma}\left(x_{k}^{e_{k}}\right)$ is disjoint from $X^{\sigma}\left(x_{1}^{e_{1}}\right)$. In particular, $X^{\sigma}\left(x_{1}^{e_{1}}\right) \neq X^{\sigma}\left(x_{k}^{e_{k}}\right)$.

This completes the proof of Theorem 5.2.

We can now prove the main theorem.

Theorem 2.2. Given a graph $\Gamma$ and a nice realization $\mathbb{X}=\left\{X_{1}, \ldots, X_{n}\right\}$ of $\Gamma$ in $S$, there exists a constant $C>0$ with the following property. If $\mathbb{F}=\left\{f_{1}, \ldots, f_{n}\right\}$ is fully supported on $\mathbb{X}$ and $\tau_{X_{i}}\left(f_{i}\right) \geq C$ for all $i=1, \ldots, n$, then the associated homomorphism

$$
\phi_{\mathbb{F}}: G(\Gamma) \rightarrow \operatorname{Mod}(S)
$$

is a quasi-isometric embedding. Furthermore, the orbit map $G \rightarrow \mathcal{T}(S)$ is a quasiisometric embedding for both $d_{\mathcal{T}}$ and $d_{\mathrm{WP}}$.

Proof. We first prove that given $\mu \in \tilde{\mathcal{M}}(S)$, we can choose $C>0$ so that if $\tau_{X_{i}}\left(f_{i}\right) \geq$ $C$ for each $i$, then the orbit map $G(\Gamma) \rightarrow \tilde{\mathcal{M}}(S)$ given by $\sigma \mapsto \phi_{\mathbb{F}}(\sigma) \mu$ is a quasiisometric embedding. Since the orbit map $\operatorname{Mod}(S) \rightarrow \widetilde{\mathcal{M}}(S)$ is a quasi-isometry, this will suffice to prove the first statement. Let $K>0$ be as in the proof of Theorem 5.2 and let $C=2 K$.

First observe that for any metric space $(\mathfrak{X}, d)$, any $x \in \mathfrak{X}$ and any $\sigma, \tau \in G(\Gamma)$, the triangle inequality implies

$$
d(\sigma \cdot x, \tau \cdot x) \leq A d_{G}(\sigma, \tau)
$$

as long as $A \geq \max \left\{d\left(s_{i} \cdot x, x\right)\right\}_{i=1}^{n}$ (here $s_{i}$ are the generators for $G$ ). 
In particular, given $\mu \in \tilde{\mathcal{M}}(S)$, to prove that the orbit map to $\tilde{\mathcal{M}}(S)$ is a quasiisometry, it suffices to find $A \geq 1$ and $B \geq 0$ so that

$$
d_{G}(1, \sigma) \leq A d_{\widetilde{M}}\left(\mu, \phi_{\mathbb{F}}(\sigma)(\mu)\right)+B
$$

for all $\sigma \in G$ (we increase $A$ if necessary so that $A \geq \max \left\{d_{\widetilde{\mathcal{M}}}\left(\mu, \phi_{\mathbb{F}}\left(s_{i}\right) \mu\right)\right\}_{i=1}^{n}$ ).

Since $K \geq K_{0}$, from Theorem 3.1 there exist $A$ and $B$ so that for all $\sigma \in G$,

$$
\sum_{X \in \Omega\left(K, \mu, \phi_{\mathbb{F}}(\sigma)(\mu)\right)} d_{X}\left(\mu, \phi_{\mathbb{F}}(\sigma)(\mu)\right) \leq A d_{\widetilde{\mathcal{M}}}\left(\mu, \phi_{\mathbb{F}}(\sigma)(\mu)\right)+B .
$$

On the other hand, if we let $w=x_{1}^{e_{1}} \ldots x_{k}^{e_{k}} \in \operatorname{Min}(\sigma)$ then by Theorem 5.2, since $\tau_{X_{i}}\left(f_{i}\right) \geq C=2 K$ (and since $K \geq 1$ ), we have

$$
\begin{aligned}
d_{G}(1, \sigma)=\sum_{i=1}^{k}\left|e_{i}\right| & \leq \sum_{i=1}^{k} K\left|e_{i}\right| \\
& \leq \sum_{i=1}^{k} d_{X^{\sigma}\left(x_{i}^{e_{i}}\right)}\left(\mu, \phi_{\mathbb{F}}(\sigma)(\mu)\right) \\
& \leq \sum_{X \in \Omega\left(K, \mu, \phi_{\mathbb{F}}(\mu)\right)} d_{X}\left(\mu, \phi_{\mathbb{F}}(\sigma)(\mu)\right) . \\
& \leq A d_{\widetilde{\mathcal{M}}}\left(\mu, \phi_{\mathbb{F}}(\sigma)(\mu)\right)+B,
\end{aligned}
$$

which completes the proof of the first statement.

The proof of the statements regarding Teichmüller space are essentially identical. For this, we observe that the topological types of the surfaces in $X^{\sigma}(\operatorname{syl}(\sigma))$ are the same as those of $\mathbb{X}$, and hence all are nonannular. That is, $X^{\sigma}(\operatorname{syl}(\sigma)) \subset$ $\Omega_{n}\left(K, \mu, \phi_{\mathbb{F}}(\mu)\right)$. So the above proof can be carried out replacing the use of Theorem 3.1 with the use of Theorems 3.2 and 3.3.

\section{Elements of the constructed subgroups}

We now assume the hypothesis of Theorem 2.2 (and hence also Theorem 5.2) on $\Gamma$, $\mathbb{X}=\left\{X_{1}, \ldots, X_{n}\right\}, C=2 K>0$, and $\mathbb{F}=\left\{f_{1}, \ldots, f_{n}\right\}$, and let

$$
\phi_{\mathbb{F}}: G(\Gamma) \rightarrow \operatorname{Mod}(S)
$$

denote the associated homomorphism. In this section we describe, in terms of the Thurston classification, the mapping class image of any $\sigma \in G(\Gamma)$. In particular, we identify all pseudo-Anosov elements in the image.

Conjugate elements in $G(\Gamma)$ map to conjugate elements in $\operatorname{Mod}(S)$, and conjugation preserves mapping class type, displacing the support of a mapping class by the homeomorphism corresponding to the conjugating element. Therefore to understand the image of $\sigma \in G(\Gamma)$, we may assume it is an element with the minimal number of 
syllables among members of its conjugacy class (there may be more than one such element, but we just pick one). We represent $\sigma$ by a word $w \in \operatorname{Min}(\sigma)$. By changing the indices if necessary, we can assume that $w$ is a word in the first $r=r(\sigma)$ generators $s_{1}, \ldots, s_{r}$, and $r$ is the least number of generators needed to write $w$ (note that a conjugate also having the minimal number of syllables will be written in terms of this same set of generators).

Remark. In what follows, we will always assume that the indices on the generators are of this type for the particular element $\sigma$ we are interested in.

We write Fill $\left(X_{1}, \ldots, X_{r}\right)$ to denote the minimal union of subsurfaces, ordered by inclusion, which contains all of the subsurfaces $X_{1}, \ldots, X_{r}$. Alternatively, Fill $\left(X_{1}, \ldots, X_{r}\right)$ is the unique union of subsurfaces containing $X_{1} \cup \cdots \cup X_{r}$ with the property that for every essential curve $\gamma$ contained in it, the projection of $\gamma$ to at least one of $X_{1}, \ldots, X_{r}$ is nontrivial. Write Fill $\mathbb{X}(\sigma)=\operatorname{Fill}\left(X_{1}, \ldots, X_{r}\right)$.

Now, if $\sigma^{\prime}=\sigma_{0} \sigma \sigma_{0}^{-1}$, then we define $\operatorname{Fill}_{\mathbb{F}}\left(\sigma^{\prime}\right)=\phi_{\mathbb{F}}\left(\sigma_{0}\right)\left(\right.$ Fill $\left._{\mathbb{X}}(\sigma)\right)$. Note that if $\sigma_{0}$ is the identity so $\sigma^{\prime}=\sigma$, then Fill $\mathbb{F}_{\mathbb{F}}(\sigma)=$ Fill $_{\mathbb{X}}(\sigma)$ depends only on $\mathbb{X}$, whereas otherwise, it may also depend on $\mathbb{F}$.

It follows easily from the discussion above that for any $\sigma, \phi_{\mathbb{F}}(\sigma)$ is supported on Fill ${ }_{\mathbb{F}}(\sigma)$. That is, $\phi_{\mathbb{F}}(\sigma)$ is represented by a homeomorphism which is the identity outside Fill $_{\mathbb{F}}(\sigma)$. In this section, we prove the following.

Theorem 6.1. Suppose that $\Gamma, \mathbb{X}=\left\{X_{1}, \ldots, X_{n}\right\}, C=2 K>0, \mathbb{F}=\left\{f_{1}, \ldots, f_{n}\right\}$ satisfy the hypotheses of Theorem 2.2, and let

$$
\phi_{\mathbb{F}}=\phi: G(\Gamma) \rightarrow \operatorname{Mod}(S)
$$

denote the associated homomorphism. Then $\phi(\sigma)$ is pseudo-Anosov on each component of Fill ${ }_{\mathbb{F}}(\sigma)$. In particular, $\phi(\sigma)$ is pseudo-Anosov if and only if Fill $_{\mathbb{F}}(\sigma)=S$.

Remark. We note that this theorem gives a sufficient condition (which may be of some independent interest) for a composition of mapping classes to be pseudo-Anosov.

Before we begin the proof, we explain a few reductions which will greatly simplify the exposition. First, as noted above, we need only consider the case that $\sigma$ has the minimal number of syllables among all its conjugates, so we assume this is the case from now on. Therefore Fill $_{\mathbb{F}}(\sigma)=$ Fill $_{\mathbb{X}}(\sigma)$.

Next, we wish to further reduce to the case that Fill $\mathbb{X}(\sigma)$ is connected. To describe this reduction, first let $\Gamma^{\prime}$ denote the subgraph spanned by $s_{1}, \ldots, s_{r}$. Since the other generators play no role in this discussion, we assume as we may that $\Gamma^{\prime}=\Gamma$. Let $\Gamma^{c}$ be the complement of $\Gamma$. That is, $\Gamma^{c}$ is the graph with the same vertex set as $\Gamma$ and where two vertices span an edge in $\Gamma^{c}$ if and only if they do not span an edge in $\Gamma$. Note that generators/vertices $s_{i}$ and $s_{j}$ in different components of $\Gamma^{c}$ commute. Therefore, we may write $\sigma=\sigma_{1} \ldots \sigma_{\ell}$, where $\ell$ is the number of components of $\Gamma^{c}$ 
and each $\sigma_{i}$ is in the group generated by vertices in a single component of $\Gamma^{c}$. In particular, $\left[\sigma_{i}, \sigma_{j}\right]=1$ for all $i$ and $j$.

Now observe that the vertices of a path in $\Gamma^{c}$ correspond to a chain of overlapping subsurfaces in $S$, and hence the components of Fill $\mathbb{X}(\sigma)$ correspond precisely to the components of $\Gamma^{c}$. In fact, one easily checks that each $\sigma_{i}$ also has the least number of syllables in its conjugacy class, and $\left\{\operatorname{Fill}_{\mathbb{X}}\left(\sigma_{1}\right), \ldots\right.$, Fill $\left._{\mathbb{X}}\left(\sigma_{\ell}\right)\right\}$ is precisely the set of components of Fill $\mathbb{X}_{\mathbb{X}}(\sigma)$. So, restricting attention to one of the subwords $\sigma_{i}$, we may assume that $\operatorname{Fill}_{\mathbb{X}}(\sigma)$ is connected.

Finally, we note that we can in fact restrict to the case that $\operatorname{Fill}_{\mathbb{X}}(\sigma)=S$. To see that this is possible, note that $\phi(G(\Gamma))$ is the identity outside $S^{\prime}=$ Fill $\mathbb{X}(\sigma)$. So, $\phi$ "restricts" to a homomorphism

$$
\widehat{\phi}: G(\Gamma) \rightarrow \operatorname{Mod}\left(S^{\prime}\right)
$$

and $\hat{\phi}(\sigma)$ is pseudo-Anosov if and only if $\phi(\sigma)$ is pseudo-Anosov on $S^{\prime}=$ Fill $\mathbb{X}(\sigma)$.

We now set out to prove Theorem 6.1 assuming (1) that $\sigma$ has the least number of syllables in its conjugacy class and (2) Fill $\mathbb{X}(\sigma)=S$. The proof makes use of the partial order on $\operatorname{syl}(\sigma)$ and $\operatorname{syl}\left(\sigma^{n}\right)$, and the order-preserving injection $X^{\sigma}(\cdot)$ of the previous section. Regarding these, let us set down a series of lemmas.

Lemma 6.2. For $\sigma$ as above, $\operatorname{Fill}\left(X^{\sigma}(\operatorname{syl}(\sigma))\right)=S$.

Proof. Fix a minimal word $x_{1}^{e_{1}} \ldots x_{k}^{e_{k}} \in \operatorname{Min}(\sigma)$. Given a curve $\gamma \subset S$, we must show that $\gamma$ intersects some $X^{\sigma}\left(x_{i}^{e_{i}}\right)=\phi\left(x_{1}^{e_{1}} \ldots x_{i-1}^{e_{i-1}}\right) X_{J(i)}$. As Fill $\mathbb{X}(\sigma)=$ Fill $\left(X_{1}, \ldots, X_{r}\right)=S$, the curve $\gamma$ intersects some $X_{j}$. Let $i$ be the minimal index such that $\gamma$ intersects $X_{J(i)}$.

If $i=1$, then $\gamma$ intersects $X_{J(1)}=X^{\sigma}\left(x_{1}^{e_{1}}\right)$. Else, $\phi\left(x_{1}^{e_{1}} \ldots x_{i-1}^{e_{i-1}}\right)(\gamma)=\gamma$. Hence $\gamma$ intersects $X^{\sigma}\left(x_{i}^{e_{i}}\right)$. As $\gamma$ was arbitrary, the lemma holds.

Lemma 6.3. For $\sigma$ as above, $w \in \operatorname{Min}(\sigma)$, and $n \in \mathbb{Z}$, we have $w^{n} \in \operatorname{Min}\left(\sigma^{n}\right)$.

Proof. Clearly $w^{n}$ represents the element $\sigma^{n}$; what needs to be shown is that this word is minimal.

Write $w=x_{1}^{e_{1}} \ldots x_{k}^{e_{k}}$ and assume that $w^{n}$ is not a minimal word representing $\sigma^{n}$. Thus we have a sequence of the three moves described in Section 4 which reduces the number of syllables in $w^{k}$. We can label the syllables of $w^{n}$ by $x_{1,1}^{e_{1}} \ldots x_{1, k}^{e_{k}} x_{2,1}^{e_{1}} \ldots x_{n, k}^{e_{k}}$ where each block $x_{j, 1}^{e_{1}} \ldots x_{j, k}^{e_{k}}=w$. As $w$ is minimal, each of the $e_{i} \neq 0$, hence in order to reduce the number of syllables of $w^{k}$ we have some sequence of type (3) moves followed by a type (2) move.

Also as $w$ is minimal, the type (2) cannot occur between syllables of the form $x_{j, i}^{e_{i}}$ and $x_{j, i^{\prime}}^{e_{i^{\prime}}}$. Therefore, after applying some type (3) moves, we have a type (2) move between syllables of the form $x_{j, i}^{e_{i}}$ and $x_{j^{\prime}, i^{\prime}}^{e^{\prime}}$ where $j<j^{\prime}$. We claim that we can assume that $j^{\prime}=j+1$. For if not, then $\left[x_{i}, x_{\ell}\right]=1$ for all $\ell$ and hence 
after a sequence of type (3) moves we could apply the move $x_{i}^{e_{i}} x_{i^{\prime}}^{e_{i^{\prime}}} \mapsto x_{i}^{e_{i}+e_{i^{\prime}}}$, contradicting the fact that $w$ is minimal.

As the set of indices $\ell$ such that $\left[x_{j, i}, x_{\ell}\right]=1$ and the set of indices $\ell$ such that $\left[x_{j^{\prime}, i^{\prime}}, x_{\ell}\right]=1$ are the same, the above assumptions give a sequence of type (3) moves on $w$ such that brings $w$ to a word of the form $x_{i^{\prime}}^{e_{i^{\prime}}} x_{1}^{e_{1}} \ldots x_{k}^{e_{k}} x_{i}^{e_{i}}$. But now conjugating $\sigma$ by $x_{i}^{e_{i}}$ results in an element with fewer syllables than $\sigma$ which contradicts our assumption that $|\operatorname{syl}(\sigma)|$ is minimal among conjugates of $\sigma$. Thus $w^{n} \in \operatorname{Min}\left(\sigma^{n}\right)$.

The above lemma allows us to define syllable shift maps $\sigma_{n}: \operatorname{syl}\left(\sigma^{n}\right) \rightarrow \operatorname{syl}\left(\sigma^{n+1}\right)$ by $\sigma_{n}\left(x_{j, i}^{e_{i}}\right)=x_{j+1, i}^{e_{i}}$ using the notation from the proof of the lemma. Notice that the maps $\sigma_{n}$ preserve the partial order. For $n>m$ we use the notation $\sigma_{m, n}=$ $\sigma_{n-1} \ldots \sigma_{m}$. The map $\sigma_{m, n}: \operatorname{syl}\left(\sigma^{m}\right) \rightarrow \operatorname{syl}\left(\sigma^{n}\right)$ shifts syllables by $n-m$ blocks and also preserves the partial order. The lemma also allows us to view $\operatorname{syl}\left(\sigma^{m}\right)$ as a subset of $\operatorname{syl}\left(\sigma^{n}\right)$, if $m \leq n$, via the obvious inclusion of $w^{m}$ as a prefix of $w^{n}$.

Under the assumption that $\operatorname{Fill}\left(X_{1}, \ldots, X_{r}\right)=S$, we have that $\Gamma^{c}$ is connected. In particular for any syllable $x_{i}^{e_{i}}$ there is another syllable $x_{j}^{e_{j}}$ such that $\left[x_{i}, x_{j}\right] \neq 1$.

Lemma 6.4. For $\sigma$ as above, and all $x_{i}^{e_{i}} \in \operatorname{syl}(\sigma)$, we have $x_{i}^{e_{i}} \prec \sigma_{1,2}\left(x_{i}^{e_{i}}\right) \in$ $\operatorname{syl}\left(\sigma^{2}\right)$.

Proof. This is similar to the proof of Lemma 6.3. If the conclusion is wrong, then $\left[x_{i}, x_{j}\right]=1$ for all syllables $x_{j}^{e_{j}} \in \operatorname{syl}(\sigma)$. This contradicts the fact that $\Gamma^{c}$ is connected.

Lemma 6.5. For $\sigma$ as above, and all $x_{i}^{e_{i}}, x_{j}^{e_{j}} \in \operatorname{syl}(\sigma)$, there exists $n, 1 \leq n \leq r+1$, such that $x_{i}^{e_{i}} \prec \sigma_{1, n}\left(x_{j}^{e_{j}}\right)$. In particular, for all syllables $x_{i}^{e_{i}}, x_{j}^{e_{j}} \in \operatorname{syl}(\sigma)$ we have $x_{i}^{e_{i}} \prec \sigma_{1, r+1}\left(x_{j}^{e_{j}}\right)$.

Proof. Fix a minimal sequence of generators $x_{i}=x_{i_{1}}, \ldots, x_{i_{m}}=x_{j}$ such that $\left[x_{i_{\ell}}, x_{i_{\ell+1}}\right] \neq 1$. Such a sequence exists as $\Gamma^{c}$ is connected. Further, notice that $m \leq r$. We will prove the lemma by induction on $m$. Specifically, we will prove that if there is a path of length $m$ between $v_{J(i)}$ and $v_{J(j)}$ in $\Gamma^{c}$, then $x_{i}^{e_{i}} \prec \sigma_{1, m+1}\left(x_{j}^{e_{j}}\right)$.

Suppose that $m=1$, hence as generators $x_{i}=x_{j}$. The case when $x_{i}^{e_{i}}=x_{j}^{e_{j}}$ as syllables in $\operatorname{syl}(\sigma)$ is covered by Lemma 6.4. Else, we must have that $x_{i}^{e_{i}} \prec x_{j}^{e_{j}}$ or $x_{j}^{e_{j}} \prec x_{i}^{e_{i}}$. In the first case using Lemma 6.4 we have $x_{i}^{e_{i}} \prec x_{j}^{e_{j}} \prec \sigma_{1,2}\left(x_{j}^{e_{j}}\right)$. In the second case, if $x_{i}^{e_{i}} \nprec \sigma_{1,2}\left(x_{j}^{e_{j}}\right)$ we can argue as in the proof of Lemma 6.3 that $|\operatorname{syl}(\sigma)|$ is not minimal among conjugates of $\sigma$.

Now by induction, we have that $x_{i}^{e_{i}} \prec \sigma_{1, m}\left(x_{i_{m-1}}^{e_{i_{m-1}}}\right)$. Since $\left[x_{i_{m-1}}, x_{j}\right] \neq$ 1 , we must have $\sigma_{1, m}\left(x_{i_{m-1}}^{e_{i_{m-1}}}\right) \prec \sigma_{1, m+1}\left(x_{j}^{e_{j}}\right)$. Hence $x_{i}^{e_{i}} \prec \sigma_{1, m}\left(x_{i_{m-1}}^{e_{i_{m-1}}}\right) \prec$ $\sigma_{1, m+1}\left(x_{j}^{e_{j}}\right)$. This completes the proof of the lemma. 
Proof of Theorem 6.1. We assume $\sigma$ is as above, so Fill $\mathbb{X}(\sigma)=S$, and prove $\phi(\sigma)$ is pseudo-Anosov. For this, it suffices to prove the following.

Claim. For every integer $N>0$ we have

$$
d_{S}\left(\partial X_{J(1)}, \phi\left(\sigma^{N(2 r+1)}\right)\left(\partial X_{J(1)}\right)\right) \geq N .
$$

Indeed, this claim says that $\phi(\sigma)$ acts with positive translation length on $\mathcal{C}(S)$ as required.

Proof of the claim. According to Lemma 6.5 we have, for any $j$,

$$
x_{1}^{e_{1}} \prec \sigma_{1, r+1}\left(x_{j}^{e_{j}}\right) \prec \sigma_{r+1,2 r+1}\left(\sigma_{1, r+1}\left(x_{1}^{e_{1}}\right)\right)=\sigma_{1,2 r+1}\left(x_{1}^{e_{1}}\right) .
$$

Now, from the definitions, we see that $X^{\sigma^{n}} \circ \sigma_{1, n}=\phi\left(\sigma^{n-1}\right) \circ X^{\sigma}$ for all $n>1$, and since $X^{\sigma}$ and $X^{\sigma^{n}}$ are order preserving by Theorem 5.2 we also have

$$
X_{J(1)} \prec \phi\left(\sigma^{r+1}\right)\left(X^{\sigma}\left(x_{j}^{e_{j}}\right)\right) \prec \phi\left(\sigma^{2 r+1}\right)\left(X_{J(1)}\right)
$$

This implies that no curve $\gamma \subset S$ is disjoint from both $\partial X_{J(1)}$ and $\phi\left(\sigma^{2 r+1}\right) \partial X_{J(1)}$. Indeed, suppose otherwise. According to Lemma 6.2, the collection of subsurfaces $\phi\left(\sigma^{r+1}\right) X^{\sigma}(\operatorname{syl}(\sigma))$ fill $S$, so there is some subsurface, say $\phi\left(\sigma^{r+1}\right) X^{\sigma}\left(x_{j}^{e_{j}}\right)$, where $\gamma$ has nonempty projection. Hence,

$$
d_{\phi\left(\sigma^{r+1}\right) X^{\sigma}\left(x_{j}^{e_{j}}\right)}\left(\partial X_{J(1)}, \phi\left(\sigma^{2 r+1}\right) \partial X_{J(1)}\right) \leq 4 .
$$

However, since $X_{J(1)} \prec \phi\left(\sigma^{r+1}\right)\left(X^{\sigma}\left(x_{j}^{e_{j}}\right)\right) \prec \phi\left(\sigma^{2 r+1}\right)\left(X_{J(1)}\right)$ it follows from Proposition 3.6 and the triangle inequality that

$$
d_{\phi\left(\sigma^{r+1}\right) X^{\sigma}\left(x_{j}^{e_{j}}\right)}\left(\partial X_{J(1)}, \phi\left(\sigma^{2 r+1}\right) \partial X_{J(1)}\right) \geq K-8>4,
$$

which is a contradiction.

By the same reasoning, no curve $\gamma$ can be disjoint from more than one of the following sets of curves

$$
\left\{\partial X_{J(1)}, \phi\left(\sigma^{2 r+1}\right)\left(\partial X_{J(1)}\right), \ldots, \phi\left(\sigma^{N(2 r+1)}\right)\left(\partial X_{J(1)}\right)\right\} .
$$

On the other hand, since $\left.X_{J(1)} \prec \phi\left(\sigma^{\ell(r+1)}\right)\left(X_{J(1)}\right)\right) \prec \phi\left(\sigma^{N(2 r+1)}\right)\left(X_{J(1)}\right)$ for all $0<\ell<N$, Proposition 3.6 and the triangle inequality again imply

$$
d_{\phi\left(\sigma^{\ell(r+1)}\right)\left(X_{J(1)}\right)}\left(\partial X_{J(1)}, \phi\left(\sigma^{N(2 r+1)}\right) \partial X_{J(1)}\right) \geq K-8 \geq K_{0},
$$

where the last inequality comes from the choice of $K$ in the proof of Theorem 5.2.

Now it follows from Theorem 3.4 that any geodesic in $\mathcal{C}(S)$ from $\partial X_{J(1)}$ to $\phi\left(\sigma^{N(2 r+1)}\right)\left(\partial X_{J(1)}\right)$ must contain a curve disjoint from each $\phi\left(\sigma^{\ell(2 r+1)}\right)\left(\partial X_{J(1)}\right)$, 
for each $\ell=0, \ldots, N$. Since these curves must all be distinct by the previous paragraph, we see that this geodesic contains at least $N+1$ vertices, so

$$
d_{S}\left(\partial X_{J(1)}, \phi\left(\sigma^{N(2 r+1)}\right)\left(\partial X_{J(1)}\right)\right) \geq N
$$

as required.

This completes the proof of the claim, and also the proof of Theorem 6.1.

\section{Surface subgroups}

In this final section we prove the following corollary of Theorem 2.2 and briefly discuss surface subgroups of right-angled Artin subgroups of the mapping class group.

Corollary 1.3. For any closed surface $S$ of genus at least 3 and any $h \geq 2$, there exist infinitely many nonconjugate genus $h$ surface subgroups of $\operatorname{Mod}(S)$, each of which acts cocompactly on some quasi-isometrically embedded hyperbolic plane in the Teichmüller space $\mathcal{T}(S)$, with either of the standard metrics.

Proof. Let $\Gamma$ be the cyclic graph of length 5 and $G(\Gamma)$ the associated right-angled Artin group. It was shown in [11] that $G(\Gamma)$ contains a quasi-isometrically embedded genus 2 surface subgroup, and hence surface subgroups of all genus $h \geq 2$ (it had been previously shown to contain a genus 5 surface subgroup in [40]). As described in [11], this example has a nice description as follows.

Suppose that the generators of $G(\Gamma)$ are $a, b, c, d, e$ with $[e, a]=[a, b]=$ $[b, c]=[c, d]=[d, e]=1$. Then the homomorphism from the fundamental group of a genus two surface to $G(\Gamma)$ is described by Figure 6 as follows. The figure shows a system of curves on the surface with labels from the set $\{a, b, c, d, e\}$ and transverse orientations. Choosing a basepoint in the complement of the curve system shown, a loop will cross the curves in the system, and one reads off an element of $G(\Gamma)$ according to the curves one crosses, and in which direction (crossing in the direction opposite the given transverse orientation, one should read an inverse of the generator); see [11] for more details.

In Section 2.4 we observed that $\Gamma$ has a nice realization $\mathbb{X}=\left\{X_{1}, \ldots, X_{5}\right\}$ in any closed surface $S$ of genus $g \geq 3$. Let $C>0$ be the constant from Theorem 2.2 and $\mathbb{F}=\left\{f_{1}, \ldots, f_{5}\right\}$ be any mapping classes fully supported on $\mathbb{X}$ with $\tau_{X_{i}}\left(f_{i}\right) \geq C$. For every $n>0$ let $\mathbb{F}^{n}=\left\{f_{1}^{n}, \ldots, f_{5}^{n}\right\}$ so that we also have $\tau_{X_{i}}\left(f_{i}^{n}\right) \geq n C$. The family of right-angled Artin subgroups $\phi_{\mathbb{F}^{n}}(G(\Gamma))$ necessarily contains infinitely many distinct conjugacy classes - observe that the proof of Theorem 2.2 implies that the minimal translation length on $\mathcal{T}(S)$ of any element of $\phi_{\mathbb{F}^{n}}(G(\Gamma))$ is tending to infinity as $n \rightarrow \infty$. Similarly, the set of surface subgroups described above, thought of as subgroups of $\operatorname{Mod}(S)$ via the homomorphisms $\phi_{\mathbb{F}^{n}}$, have minimal translation length on $\mathcal{T}(S)$ tending to infinity as $n \rightarrow \infty$. Consequently, there are infinitely many pairwise nonconjugate genus $h$ surface subgroups. 


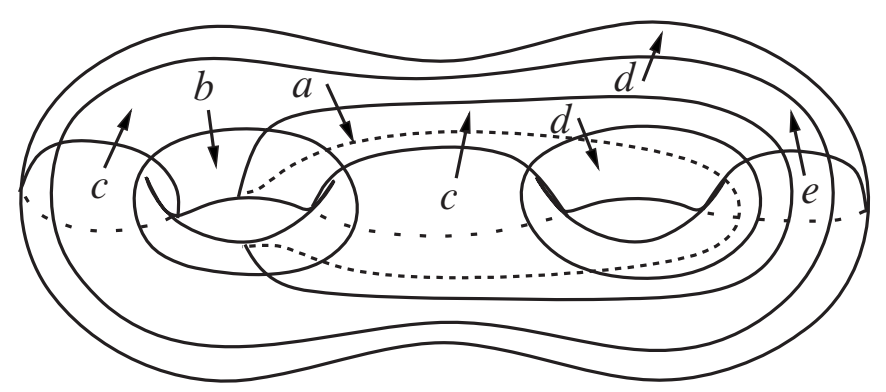

Figure 6. A curve system on a genus 2 surface which defines an embedding into $G(\Gamma)$, where $\Gamma$ is the cyclic graph of length 5 .

That each of these stabilizes a quasi-isometrically embedded hyperbolic plane $\mathbb{H} \subset \mathcal{T}(S)$ follows from the fact that the surface group itself is quasi-isometric to $\mathbb{H}$, and the orbit map defines a quasi-isometric embedding by Theorem 2.2. The surface group clearly acts cocompactly on this plane.

It follows that these surface groups all have positive translation length on Teichmüller space. However, as we have already mentioned, they cannot be purely pseudo-Anosov. In fact, for surface subgroups of right-angled Artin groups, this is always the case.

Proposition 7.1. Suppose that $G(\Gamma)<\operatorname{Mod}(S)$ is a right-angled Artin subgroup and $\pi_{1}(\Sigma)<G(\Gamma)$ is a surface subgroup. Then as a subgroup of $\operatorname{Mod}(S), \pi_{1}(\Sigma)$ contains a nontrivial reducible element.

Proof. As was shown in [11], every homomorphism from a surface group $\pi_{1}(\Sigma)$ into a right-angled Artin group $G(\Gamma)$ arises as in the proof of the previous corollary. That is, there is a curve system on $\Sigma$, each curve is endowed with a transverse orientation, the components are labeled by generators of $G(\Gamma)$, and the homomorphism is obtained by taking a loop and reading off the generators as one crosses the curves in the system.

Furthermore, one may assume that each of the curves in the system is essential, and if the homomorphism $H \rightarrow G(\Gamma)$ is injective then these curves cut $\Sigma$ into disks. Now consider a loop $\gamma$ which runs parallel to, without crossing, one of the curves in the system. Call this curve $\eta_{1}$ and suppose the associated generator of $G(\Gamma)$ is $s_{1}$. The loop $\gamma$ crosses other curves $\eta_{i_{1}}, \ldots, \eta_{i_{k}}$ and so determines some word $s_{i_{1}}^{ \pm 1} \ldots s_{i_{k}}^{ \pm 1}$, which is the image of $\gamma$ in $G(\Gamma)$. Choosing $\gamma$ to run very close to $\eta_{1}$, we can assume that the curves $\eta_{i_{1}}, \ldots, \eta_{i_{k}}$ which $\gamma$ crosses are also nontrivially intersected by $\eta_{1}$. As noted in [11], each of the associated generators $s_{i_{1}}, \ldots, s_{i_{k}}$ must commute with $s_{1}$, and be different from it.

Now we are essentially done. The image of $\gamma$ is an element which commutes with $s_{1}$, and in fact, the image of $\gamma$ and $s_{1}$ in $G(\Gamma)$ generate a subgroup isomorphic to $\mathbb{Z}^{2}$. 
If $G(\Gamma)<\operatorname{Mod}(S)$, then the image of $\gamma$ in $\operatorname{Mod}(S)$ has centralizer which contains $\mathbb{Z}^{2}$. As is well known, the image of $\gamma$ cannot be pseudo-Anosov; see [21].

Remark. In fact, the assumption that $\pi_{1}(\Sigma)$ is a surface group can be relaxed considerably. Indeed, a similar proof shows that any finitely presented 1-ended subgroup of a right-angled Artin group $G(\Gamma)<\operatorname{Mod}(S)$ contains a reducible element.

In [25], Koberda observes that $\operatorname{Mod}\left(S_{g}\right)$ is not commensurable with a right-angled Artin group if $g \geq 3$ (in fact, he proves the stronger statement that $\operatorname{Mod}\left(S_{g}\right)$ cannot virtually embed in a right-angled Artin group). This is also true for genus 2 as the following shows.

Proposition 7.2. The group $\operatorname{Mod}\left(S_{2}\right)$ is not commensurable with a right-angled Artin group.

Proof. Suppose that $\operatorname{Mod}\left(S_{2}\right)$ is commensurable with $G(\Gamma)$, with $\Lambda$ isomorphic to a finite index subgroup of both. Let $\pi_{1}(\Sigma)<\operatorname{Mod}\left(S_{2}\right)$ be a surface subgroup as constructed in [27]. In this surface group, there is exactly one element of $\pi_{1}(\Sigma)$, up to conjugacy and powers, which is not pseudo-Anosov. Moreover, this one element represents a simple closed curve $\alpha$ on $\Sigma$.

Now, $\pi_{1}(\Sigma) \cap \Lambda$ is a finite index subgroup of $\pi_{1}(\Sigma)$ and so corresponds to a covering space $p: \widetilde{\Sigma} \rightarrow \Sigma$, and we write $\pi_{1}(\tilde{\Sigma})<\pi_{1}(\Sigma)$ for the image under $p_{*}$. Note that the reducible elements of $\pi_{1}(\widetilde{\Sigma})$ in $\operatorname{Mod}\left(S_{2}\right)$ represent a finite set of pairwise disjoint simple closed curves on $\widetilde{\Sigma}$, namely $p^{-1}(\alpha)$.

On the other hand, a closer inspection of the proof of the previous proposition shows that, viewing $\Lambda<G(\Gamma)$, there are actually two elements $\gamma_{1}, \gamma_{2} \in \pi_{1}(\widetilde{\Sigma})$ which represent curves on $\widetilde{\Sigma}$ which nontrivially intersect, whose centralizers in $G(\Gamma)$ contain $\mathbb{Z}^{2}$. These must represent reducible elements in $\operatorname{Mod}(S)$, and this is a contradiction.

Remark. This same proof also works to show that the mapping class group of an $n$-punctured sphere, with $n \geq 6$, is not commensurable with any right-angled Artin group. The point is that the examples from [27] can be chosen to descend to the quotient by the hyper-elliptic involution, and then one of the punctures can be erased (with the exception of the genus 2 case).

\section{References}

[1] M. F. Atiyah, The signature of fibre-bundles. In Global Analysis (Papers in Honor of K. Kodaira), Univ. Tokyo Press, Tokyo 1969, 73-84. Zbl 0193.52302 MR 0254864

[2] J.A. Behrstock, Asymptotic geometry of the mapping class group and Teichmüller space. Geom. Topol. 10 (2006), 1523-1578. Zbl 1145.57016 MR 2255505 
[3] J. Behrstock, B. Kleiner, Y. Minsky, and L. Mosher, Geometry and rigidity of mapping class groups. Preprint 2008. arXiv:0801.2006

[4] J. Behrstock and Y. Minsky, Centroids and the rapid decay property in mapping class groups. J. London Math. Soc. (2) 84 (2011), 765-784. Zbl 05987716 MR

[5] B. H. Bowditch, Atoroidal surface bundles over surfaces. Geom. Funct. Anal. 19 (2009), 943-988. Zbl 1195.20045 MR 2570310

[6] J. F. Brock, The Weil-Petersson metric and volumes of 3-dimensional hyperbolic convex cores. J. Amer. Math. Soc. 16 (2003), 495-535. Zbl 1059.30036 MR 1969203

[7] R. Charney, An introduction to right-angled Artin groups. Geom. Dedicata 125 (2007), 141-158. Zbl 1152.20031 MR 2322545

[8] J. Crisp and B. Farb, The prevalence of surface subgroups in mapping class groups. In preparation.

[9] J. Crisp and L. Paris, The solution to a conjecture of Tits on the subgroup generated by the squares of the generators of an Artin group. Invent. Math. 145 (2001), 19-36. Zbl 1002.20021 MR 1839284

[10] J. Crisp, M. Sageev, and M. Sapir, Surface subgroups of right-angled Artin groups. Internat. J. Algebra Comput. 18 (2008), 443-491. Zbl 1193.20043 MR 2422070

[11] J. Crisp and B. Wiest, Embeddings of graph braid and surface groups in right-angled Artin groups and braid groups. Algebr. Geom. Topol. 4 (2004), 439-472. Zbl 1057.20028 MR 2077673

[12] J. Crisp and B. Wiest, Quasi-isometrically embedded subgroups of braid and diffeomorphism groups. Trans. Amer. Math. Soc. 359 (2007), 5485-5503. Zbl 1186.20027 MR 2327038

[13] B. Farb and L. Mosher, Convex cocompact subgroups of mapping class groups. Geom. Topol. 6 (2002), 91-152. Zbl 1021.20034 MR 1914566

[14] K. Fujiwara, Subgroups generated by two pseudo-Anosov elements in a mapping class group. I. Uniform exponential growth. In Groups of diffeomorphisms, Adv. Stud. Pure Math. 52, Math. Soc. Japan, Tokyo 2008, 283-296. Zbl 1170.57017 MR 2509713

[15] G. González-Díez and W. J. Harvey, Surface groups inside mapping class groups. Topology 38 (1999), 57-69. Zbl 0927.57013 MR 1644059

[16] C. M. Gordon, D. D. Long, and A. W. Reid, Surface subgroups of Coxeter and Artin groups. J. Pure Appl. Algebra 189 (2004), 135-148. Zbl 1057.20031 MR 2038569

[17] E. R. Green, Graph products of groups. PhD thesis, The University of Leeds, 1990. http://etheses.whiterose.ac.uk/236/

[18] U. Hamenstädt. Word hyperbolic extensions of surface groups. Preprint 2005. arXiv:math/0505244

[19] S. Hermiller and J. Meier, Algorithms and geometry for graph products of groups. $J$. Algebra 171 (1995), 230-257. Zbl 0831.20032 MR 1314099

[20] T. Hsu and D. T. Wise, On linear and residual properties of graph products. Michigan Math. J. 46 (1999), 251-259. Zbl 0962.20016 MR 1704150

[21] N. V. Ivanov, Subgroups of Teichmüller modular groups. Transl. Math. Monogr. 115, Amer. Math. Soc., Providence, RI, 1992 Zbl 0776.57001 MR 1195787 
[22] R. P. Kent, IV and C. J. Leininger, Shadows of mapping class groups: capturing convex cocompactness. Geom. Funct. Anal. 18 (2008), 1270-1325. Zbl 05508776 MR 2465691

[23] S.-h. Kim, Co-contractions of graphs and right-angled Artin groups. Algebr. Geom. Topol. 8 (2008), 849-868. Zbl 1143.20023 MR 2443098

[24] S.-h. Kim, On right-angled Artin groups without surface subgroups. Groups Geom. Dyn. 4 (2010), 275-307. Zbl 05696866 MR 2595093

[25] T. Koberda, Right-angled Artin groups and a generalized isomorphism problem for finitely generated subgroups of mapping class groups. Preprint 2010. arXiv:1007.1118

[26] K. Kodaira, A certain type of irregular algebraic surfaces. J. Analyse Math. 19 (1967), 207-215. Zbl 0172.37901 MR 0216521

[27] C. J. Leininger and A. W. Reid, A combination theorem for Veech subgroups of the mapping class group. Geom. Funct. Anal. 16 (2006), 403-436. Zbl 1099.57002 MR 2231468

[28] M. Linch, A comparison of metrics on Teichmüller space. Proc. Amer. Math. Soc. 43 (1974), 349-352. Zbl 0284.32015 MR 0338453

[29] K. Liu, X. Sun, and S.-T. Yau, Canonical metrics on the moduli space of Riemann surfaces I. J. Differential Geom. 68 (2004), 571-637. Zbl 1078.30038 MR 2144543

[30] K. Liu, X. Sun, and S.-T. Yau, Canonical metrics on the moduli space of Riemann surfaces II. J. Differential Geom. 69 (2005), 163-216. Zbl 1086.32011 MR 2169586

[31] D. D. Long, A. W. Reid, and M. Thistlethwaite, Zariski dense surface subgroups in SL $(3, \mathbf{Z})$. Preprint 2010.

[32] J. Mangahas, Uniform uniform exponential growth of subgroups of the mapping class group. Geom. Funct. Anal. 19 (2010), 1468-1480. Zbl 1207.57005 MR 2585580

[33] H. A. Masur and Y. N. Minsky, Geometry of the complex of curves I: Hyperbolicity. Invent. Math. 138 (1999), 103-149. Zbl 0941.32012 MR 1714338

[34] H. A. Masur and Y. N. Minsky, Geometry of the complex of curves II: Hierarchical structure. Geom. Funct. Anal. 10 (2000), 902-974. Zbl 0972.32011 MR 1791145

[35] J. McCarthy, A "Tits-alternative" for subgroups of surface mapping class groups. Trans. Amer. Math. Soc. 291 (1985), 583-612. Zbl 0579.57006 MR 800253

[36] C. T. McMullen, The moduli space of Riemann surfaces is Kähler hyperbolic. Ann. of Math. (2) 151 (2000), 327-357. Zbl 0988.32012 MR 1745010

[37] L. Mosher, A hyperbolic-by-hyperbolic hyperbolic group. Proc. Amer. Math. Soc. 125 (1997), 3447-3455. Zbl 0895.20028 MR 1443845

[38] K. Rafi, A combinatorial model for the Teichmüller metric. Geom. Funct. Anal. 17 (2007), 936-959. Zbl 1129.30031 MR 2346280

[39] C. E. Röver, On subgroups of the pentagon group. Math. Proc. Roy. Irish Acad. 107 (2007), 11-13. Zbl 1121.57003 MR 2289796

[40] H. Servatius, C. Droms, and B. Servatius, Surface subgroups of graph groups. Proc. Amer. Math. Soc. 106 (1989), 573-578. Zbl 0677.20023 MR 952322

[41] S. Wang, Representations of surface groups and right-angled Artin groups in higher rank. Algebr. Geom. Topol. 7 (2007), 1099-1117. Zbl 1133.20024 MR 2336251 
[42] S.-K. Yeung, Quasi-isometry of metrics on Teichmüller spaces. Internat. Math. Res. Notices 2005 (2005), 239-255. Zbl 1069.32005 MR 2128436

Received June 23, 2010; revised December 30, 2010

M. T. Clay, Department of Mathematics, Allegheny College, Meadville, PA 16335, U.S.A.

E-mail: mclay@allegheny.edu

C. J. Leininger, Department of Mathematics, University of Illinois at Urbana-Champaign, Urbana, IL 61801, U.S.A.

E-mail: clein@math.uiuc.edu

J. Mangahas, Mathematics Department, Brown University, Providence, RI 02912, U.S.A.

E-mail: mangahas@math.brown.edu 